\title{
Oxidation of Toluene in Humid Air by Metal Oxides Supported on
}

\section{$\gamma$-Alumina}

Mohammadreza Esmaeilirad ${ }^{\mathrm{a}}$, Mohammad Zabihi ${ }^{\mathrm{b}}$ *, Jalal Shayegan ${ }^{\mathrm{a}}$, and Farhad Khorasheh ${ }^{\mathrm{a}}$

${ }^{a}$ Department of Chemical and Petroleum Engineering, Sharif University of Technology, Azadi Avenue, P.O. Box 11155-9465, Tehran, Iran.

${ }^{\mathrm{b}}$ Chemical Engineering Faculty, Sahand University of Technology, P.O. Box 51335-1996, Sahand New Town, Tabriz, Iran.

*Corresponding author, E-mail: zabihi@ sut.ac.ir 


\begin{abstract}
Monometallic and bimetallic supported metal oxides catalysts on $\gamma$-alumina were prepared by heterogeneous deposition-precipitation. The $\gamma$-alumina used as a support was synthesized by the sol-gel and the co-precipitation methods. Supports and catalysts were characterized by Brunauer-Emmett-Teller (BET) surface area, X-ray diffraction (XRD), field-emission scanning electron microscopy (FESEM), transmission electron microscopy (TEM), and X-ray photoelectron spectroscopy (XPS). The performance of the prepared catalysts was studied for total oxidation of toluene in air at different relative humidity and oxidation temperatures. Efficiency of bimetallic catalysts for deep oxidation of toluene was higher than copper oxide supported on $\gamma$-alumina. Although increasing the lanthanum, cobalt, and nickel loading on the support led to a modified catalyst surface and morphology, the catalytic activity of bimetallic catalysts decreased with increasing lanthanum, cobalt, and nickel content due to the reduced amount of copper oxide which has a higher activity for oxidation of volatile organic compounds. The $\gamma$-alumina prepared by the sol-gel method using ethanol as a solvent (AlSE) was the best support and La-Cu/AlSE had the best performance (toluene removal efficiency $>90 \%$ ). In addition, the presence of water vapor in the feed had a negative effect on toluene conversion.
\end{abstract}

Key Words: Toluene, Oxidation, $\gamma$-alumina, Sol-gel, Co-precipitation, Heterogeneous deposition-precipitation, Bimetallic catalyst. 


\section{Introduction}

Numerous studies have reported that catalytic oxidation is an efficient and feasible technique for removal of volatile organic compounds (VOCs) from air and the complete oxidation of toluene as a model VOC in air has been widely reported in the literature [1]. Only a few studies have reported the influence of water vapor in the feed on the catalytic oxidation of VOCs [2-3]. The effects of water vapor on the performance of supported catalysts depend on the nature and morphology of the catalysts. Studies reported in the open literature suggest the negative effect of feed humidity on the catalyst activity. Water vapor is an end product in the oxidation of VOCs and could also be present in a humid feed [4]. It has been reported that the conversion of toluene decreased with increasing relative humidity in the feed due to the competition between water vapor, carbon dioxide, and toluene for adsorption on the metal oxides [5-7]. There are a number of investigations on the oxidation of toluene over supported monometallic and bimetallic metal oxides catalysts with transition and noble metals using a variety of supports including alumina [8-9], carbonaceous materials such as activated carbon [1] and carbon nano tubes [10], halloysite [11], and $\mathrm{CeO}_{2}[12]$. Nano structural alumina with high surface area and high stability is an appropriate low cost support for deep oxidation of toluene [13-14]. Sol-gel and co-precipitation are the most common methods to prepare different forms of alumina including $\gamma$ and $\alpha$-alumina powders [15-16]. Alumina particles were synthesized with various shapes and sizes depending on the preparation method. Hexagonal and spherical alumina particles were prepared by the co-precipitation method while spherical alumina particles were formed by the sol-gel method [17]. In a study where $\gamma$ alumina was synthesized by the controlled precipitation method using different precipitating agents, it was found that $\gamma$-alumina made from ammonium bicarbonate had higher surface area and adsorption capacity [18].Although there are extensive studies reported in the literature on the deep oxidation of hydrocarbons by supported bimetallic catalysts [1], no studies were detected in the open literature which had examined the effects of the alumina support 
preparation method on the catalyst performance or the performance supported metal oxides catalyst prepared by heterogeneous deposition-precipitation over $\gamma$-alumina.

The main scope of this investigation was to prepare nano dispersed supported monometallic and bimetallic catalysts on $\gamma$-alumina synthesized by sol-gel and coprecipitation methods and examine the effect of the support preparation method on the catalyst performance. Heterogeneous deposition-precipitation was employed for preparing bimetallic catalysts with copper, lanthanum, cobalt, and nickel oxides. The influence of the relative humidity of the feed and the oxidation temperature was also $\_$in the oxidation of toluene in contaminated air over the prepared catalysts.

\section{Experimental section}

\subsection{Support Preparation}

All chemicals and reagents were obtained from Merck. The following starting materials were used in the co-precipitation method for preparation of nano-alumina: aluminum nitrate $\left(\mathrm{Al}\left(\mathrm{NO}_{3}\right)_{3} \cdot 9 \mathrm{H}_{2} \mathrm{O}, 1.1 \mathrm{M}\right)$, ammonium nitrate $\left(\mathrm{NH}_{4} \mathrm{NO}_{3}, 1.4 \mathrm{M}\right)$, sodium bicarbonate $\left(\mathrm{NaHCO}_{3}\right)$, and ethanol $\left(\mathrm{C}_{2} \mathrm{H}_{6} \mathrm{OH}, 75\right.$ wt.\% $)$. Co-precipitation was carried out at in a batch system at constant $\mathrm{pH}$ using a mixture containing specified amounts of aluminumnitrate and ammonium nitrate. Sodium bicarbonate was added dropwise to mixture which was kept at $70^{\circ} \mathrm{C}$ to maintain the $\mathrm{pH}$ at 8 . Precipitation was continued for 3 hours. The precipitate was filtrated, washed by ethanol, and subsequently dried at ambient temperature overnight. The dried sample was then calcined under nitrogen flow at $550^{\circ} \mathrm{C}$ for 5 hours. In the sol-gel method, a $0.1 \mathrm{M}\left(\mathrm{C}_{4} \mathrm{H}_{9}\right)_{3} \mathrm{AlO}_{3}$ solution was prepared with ethanol and heated at $90^{\circ} \mathrm{C}$. An ammonia solution (28 wt.\%) was then added until the gel appeared in the solution. Aging was conducted at room temperature for one day and the gel was subsequently dried at $100^{\circ} \mathrm{C}$ for 24 hours. Calcination was carried out by heating the sample under nitrogen flow to $1000^{\circ} \mathrm{C}$ at a rate of $20^{\circ} \mathrm{C} / \mathrm{min}$ and maintaining it at $1000^{\circ} \mathrm{C}$ for 2 hours. The sol-gel method was repeated using methanol (100 wt. \%) as a solvent. The three alumina samples were named 
AlSE (sol-gel with ethanol), AlSM (sol-gel with methanol), AlCP (co-precipitation with ethanol).

\subsection{Catalyst Preparation}

Supported monometallic and bimetallic catalysts on nano-alumina were prepared by the deposition-precipitation method. Nitrate solutions of metals (copper, cobalt, lanthanum, and nickel) were prepared by addition of a specified volume of deionized water to the metal salts. Deposition-precipitation was conducted for 9 hours with mixing and heating at $70-80^{\circ} \mathrm{C}$ and metal ions were precipitated on the sieved nano-alumina (below mesh 60) by adding $\mathrm{NaOH}$ solution (6 wt.\%) in 30 minute intervals until reaching a basic solution of $\mathrm{pH}=10-11$ to obtain an 8 wt.\% metal loading. The solution was filtered and the catalyst sample was washed by heated deionized water several times. The sample was then dried in an oven at $110^{\circ} \mathrm{C}$ overnight. The dried catalyst was subsequently calcined in a tubular furnace under nitrogen flow for 3 hours at $550^{\circ} \mathrm{C}$. Preparation of bimetallic catalysts with a total of 8 wt. $\%$ metal loading with various ratios (Metal: $\mathrm{Cu}=1: 1,1: 3$ and 3:1) were carried out by co-depositionprecipitation with the nitrate solutions of metals being added simultaneously to the sieved nano-alumina support. Prepared catalysts were denoted by types of support and metal content, Metal (x(wt. \%) $) \mathrm{Cu}(\mathrm{y}($ wt. \%) $) /$ Support, $(\mathrm{x}=2,4$ and 6, $\mathrm{y}=8-\mathrm{x})$. For example, the bimetallic catalyst with 2 wt. \% $\mathrm{Cu}$ and 6 wt. \% La supported on nano-alumina prepared by the sol-gel method with ethanol was named La6-Cu2/A1SE.

\subsection{Catalyst Activity}

The schematic of the experimental apparatus to carry out the oxidation of toluene in air in the presence of water vapor is presented in Fig. 1. The performance of the prepared catalysts was evaluated in a tubular fixed bed reactor operating at atmospheric pressure. Inlet concentration of toluene and relative humidity were controlled by allowing an air stream to 
pass at a specified flow rate set with a rotameter over isolated bubbling saturators each filled with toluene and water. Thermocouples placed in each saturator were used to monitor the liquid temperature in each saturator to control the liquid temperature to provide the desired concentration of toluene and water vapor in the feed to the reactor. A digital thermocouple located at the center inside the reactor and a PID temperature controller was used to monitor and maintain the reactor temperature at the desired level. $1 \mathrm{~g}$ of catalyst was packed in the center of the horizontal quartz reactor that was $10 \mathrm{~cm}$ in length with an internal diameter of 12 $\mathrm{mm}$. Glass beads and glass wool were used to pack the reactor to prevent flow channeling. The oxidation temperature and relative humidity were adjusted in the range of $250-450^{\circ} \mathrm{C}$ and 0-94\%, respectively. Outlet concentrations from the reactor were determined by gas chromatography-mass spectroscopy (GC-Mass, Agilent) equipped with a 5975C mass detector and $30 \mathrm{~m}$ long HP-5MS stainless steel column. The relative humidity of the feed was measured by an electrical thermometer and humidity meter (humidifier, ST-625) at regular time intervals. There were no intermediate oxidation products at the outlet of the reactor indicating the complete oxidation of toluene. All experiments were performed at a constant feed flow rate of $166 \mathrm{ml} / \mathrm{min}$ with inlet toluene concentration of $10000 \mathrm{ppmv}$.

\subsection{Catalyst Characterization}

Surface area and pore sizes of catalysts were determined by nitrogen adsorption/desorption at $77 \mathrm{~K}$ using a BEL (Japan) physical-adsorption apparatus. Catalyst surface area and average pore size was measured by Brunauer-Emmett-Teller (BET) theory. $\mathrm{X}$-ray Diffraction (XRD) patterns obtained at $30 \mathrm{kV}, 20 \mathrm{~mA}$, and copper $\mathrm{K} \alpha$ radiation at scanning rate of $3 \%$ min using a STOE model D-64295equipment were used for identification and determination of the size of catalyst crystallites. The surface morphologies of supported monometallic and bimetallic catalysts were examined using a field-emission electron microscope (FESEM) at $15 \mathrm{kV}$ using a model S-4160 Hitachi equipment from Japan. Micro 
images of supported catalysts were obtained by transmission electron microscopy (TEM) to investigate the morphology and size distribution of the prepared catalyst samples using an EM208-Philips instrument equipped with a Schottky field emission gun operated at $100 \mathrm{keV}$ and magnification range of $180000 x$. XPS analysis was carried out on AXIS Ultra DLD (Kratos Analytical, UK).

\section{Results and Discussion}

\subsection{Support Characterization}

XRD patterns of different supports prepared by co-precipitation and sol-gel methods are presented in Fig. 2. Different alumina phases might be formed during the preparation steps depending on the growth temperatures. Boehmite phase can be observed at low temperatures. $\gamma$-Alumina with a high surface area and a high thermal stability would appear with increasing

growth temperature. The $\alpha$-alumina phase with tetragonal morphology and having a higher thermal stability and lower surface area than $\gamma$-alumina requires high growth temperatures $(\sim$ $1000^{\circ} \mathrm{C}$ ). Consequently, $\gamma$-alumina with high surface area can be a very suitable catalyst support. As indicated in Fig. 2, the sol-gel method is an appropriate method for synthesis of $\gamma$ alumina with smaller crystallite sizes. The size of the crystallites was obtained using Scherrer equation:

$$
D=\frac{K \lambda}{\beta \cos \theta}
$$

Where $\mathrm{D}$ is the mean crystallite diameter $(\mathrm{nm}), \mathrm{K}$ is the dimensionless shape factor(spheres = 0.89 ), $\lambda$ is the $\mathrm{X}$-ray wavelength (X-ray tube: $\mathrm{Cu}, \lambda=0.154 \mathrm{~nm}$ ), $\beta$ is the line broadening at half the maximum intensity and $\theta$ is the Bragg angle. The crystallite sizes for different alumina supports are reported in Table 1 indicating that the sol-gel method with ethanol as solvent is an efficient and the feasible preparation technique to generate well dispersed nano alumina as compared with the co-precipitation method. 
FESEM images of the prepared supports are presented in Fig. 3. Alumina showed homogenous sphere structure with light color in all images. The images indicate that alumina prepared by the sol-gel method using ethanol as a solvent has a more homogenous structure with higher dispersion of particles. These results are in agreement with the XRD patterns. The observed particle sizes from FESEM images also confirm the results obtained from Scherrer equation.

Architectural porosity of three types of gamma-alumina (AlCP, AlSE and AlSM) was studied by $\mathrm{N}_{2}$ adsorption-desorption test, which showed type-IV isotherm that is typical for mesoporous adsorbents shown in Fig. 4 based on the IUPAC classification. Important feature of type IV isotherms is the presence of micropores associated with mesopores. It can provide an appropriate porous media for metal ions during catalyst preparation method. The surface area for the AlCP, AlSE and AlSE is calculated 305, 284 and $97 \mathrm{~m}^{2} / \mathrm{g}$ by BET theory, respectively. It is shown the surface area decreased by forming alumina by sol-gel method with methanol as a solvent which is confirming the XRD and FESEM Results.

The surface area and total pore volume AISE, AlSM and AlCP obtained by BET, BJH and t-plot analyses are summarized in Table 2.

\subsection{Characterization of Monometallic Catalysts}

The XRD patterns of monometallic catalysts Cu8/A1SE, Cu8/AlCP, and Cu8/A1SM are presented in Fig.5. Broad peaks with high intensity corresponding to crystallites of $\mathrm{CuO}$ were detected at $2 \theta=33-40^{\circ}$ (ICDD: $\left.00-001-1117\right) . \mathrm{Cu}(\mathrm{OH})_{2}$ that was formed during catalyst preparation by heterogeneous deposition-precipitation, resulted in the formation copper oxide during the calcinations step that was carried out at high temperatures [1] .XRD patterns did not indicate the formation of $\mathrm{Cu}_{2} \mathrm{O}$ and $\mathrm{Cu}_{3} \mathrm{O}_{4}$ Over $\gamma$-alumina as was checked by (00-0330480) and (00-001-1142) ICDD codes representative of $\mathrm{Cu}_{3} \mathrm{O}_{4}$ and $\mathrm{Cu}_{2} \mathrm{O}$, respectively. The sizes of crystallites were calculated to be below $9 \mathrm{~nm}$ according to Scherrer equation and are 
reported in Table 3. Results demonstrated that well dispersed nano-structural supported copper oxide catalysts were synthesized when the $\gamma$-alumina was prepared by thesol-gel method with ethanol as a solvent.

FESEM images were obtained for Cu8/AlSE, Cu8/AlCP, and Cu8/AlSM catalysts and are presented in Fig. 6 all indicating the bright spherical morphology of nano-particles with high dispersion over $\gamma$-alumina. The best results were obtained for Cu8/AlSE with smallest dispersed particles in agreement with XRD results.

Fig. 7 illustrates the TEM images of Cu8/AlSE which was a highly dispersed supported copper oxide over $\gamma$-alumina in accordance with XRD and FESEM results. The darker and spherical dots represent $\mathrm{CuO}$ crystallites over $\gamma$-alumina. Fig. 7 also indicates that size of the uniform $\mathrm{CuO}$ crystallites were smaller than $9 \mathrm{~nm}$. The size distributions of the crystallites were obtained by Clemex vision software.

Nitrogen adsorption/desorption isotherms for Cu8/AlSE, Cu8/AlCP, and Cu8/AlSM are presented in Fig. 8 indicating a type IV isotherm with hysteresis loops for adsorption on the mesoporous $\gamma$-alumina support synthesized by the sol-gel method. Fig. 8 demonstrates the high capacity of Cu8/AlSE for adsorption of nitrogen. Porosity, average pore diameter, and the specific surface area of the catalysts are reported in Table 4. Crystallites with larger size and lower surface area were generated for Cu8/AlSM due to the longer nucleation time in the sol-gel method with methanol as a solvent.

\subsection{Characterization of Bimetallic Catalysts}

XRD patterns for three types of copper, nickel, cobalt, and lanthanum bimetallic catalysts supported over $\gamma$-alumina by heterogeneous deposition-precipitation are presented in Fig. 9. XRD patterns of La6-Cu2/A1SE, La4-Cu4/A1SE and La2-Cu6/AlSEin Fig. 9a indicate two types of crystallites generated over alumina. XRD patterns were compared with those for 
$\mathrm{CuO}, \mathrm{LaO}, \mathrm{LaCu}_{6}, \mathrm{La}_{2} \mathrm{O}_{3}, \mathrm{Al}_{2} \mathrm{La}, \mathrm{CuLaO}_{2}$, and $\mathrm{CuLa}_{2} \mathrm{O}_{4}$ from ICDD: 00-080-1917, 00-0180680, 00-037-1448, 01-083-1345, 00-003-0699, 00-022-0238, and 00-022-0239, respectively. The patterns indicated that the strong and narrow peaks at $2 \theta=35,53$, and $38^{\circ}$ corresponded to $\mathrm{CuO}, \mathrm{LaO}$, and $\mathrm{LaCu}_{6}$, respectively. There were no detected crystallite phases for $\mathrm{La}_{2} \mathrm{O}_{3}$, $\mathrm{Al}_{2} \mathrm{La}, \mathrm{CuLaO}_{2}$ and $\mathrm{CuLa}_{2} \mathrm{O}_{4}$ from the $\mathrm{XRD}$ patterns. The broad peaks with low intensity disappeared with increasing La loading on the support due to its high adsorption tendency compared with copper ions suggesting that La is initially adsorbed on the support and the available surface would provide for a higher dispersion of copper over the alumina support. The XRD patterns for Co2-Cu6/AlSE, Co4-Cu4/AlSE, and Co6-Cu2/AlSE are presented in Fig. 9b. Similar results were obtained by loading cobalt and copper ions over alumina simultaneously. As the adsorption tendency for cobalt ions is higher than copper ions on alumina, cobalt ions are dominant in contrast with copper ions for adsorption on alumina. The broad and narrow peaks appeared with increasing cobalt content and $\mathrm{CoO}$ crystallites were generated instead of $\mathrm{Co}_{2} \mathrm{O}_{3}$ crystallites. Strong peaks at $2 \theta=46-61^{\circ}, 31^{\circ}$, and $35-38^{\circ}$ represent $\mathrm{CoO}, \mathrm{Co}_{2} \mathrm{O}_{3}$, and $\mathrm{Co}_{2} \mathrm{O}_{3}$ crystallites, respectively. No peaks were detected for $\mathrm{Co}_{2} \mathrm{O}_{3} \mathrm{CuO}$, $\mathrm{Co}$ and $\mathrm{Al}_{62} \mathrm{Cu}_{20} \mathrm{Co}_{15}$ over alumina. The XRD patterns for three different catalysts synthesized by copper and nickel deposition are presented in Fig. 9c. The results illustrate that better dispersed supported bimetallic catalysts were generated with increasing nickel and decreasing copper loading over the nano-alumina support. Detectable crystallites for this type of catalysts consisted of $\mathrm{NiO}, \mathrm{NiAl}_{2} \mathrm{O}_{4}, \mathrm{Ni}_{2} \mathrm{O}_{3}$ and $\mathrm{CuO}$ at $2 \theta=43-63^{\circ}, 37^{\circ}, 31-33^{\circ}$, and $35-38^{\circ}$ in the agreement with ICDD codes00-001-1239, 00010-0339, 00-010-0339, and 01-080-1917, respectively. XRD peaks were examined for the presence of $\mathrm{Cu}_{0.8} \mathrm{Ni}$ according to ICDD code (00-009-0205) which was undetectable. With increasing nickel content peaks with lower intensity and broader width were observed implying that nickel was more readily deposited on the alumina surface compared with copper. The crystallite sizes for these three groups of bimetallic catalysts were calculated by Scherrer equation and are reported in Table 5 with the 
smallest size belonging to $\mathrm{La}-\mathrm{Cu} / \mathrm{AlSE}$ catalysts. There were no significant differences between the crystallite sizes of Ni-Cu/AlSE and Co-Cu/AlSE catalysts.

TEM images of some of the bimetallic supported catalysts with different copper, cobalt, nickel, and lanthanum contents are presented in Fig. 10 indicating three different colors including white, lighter black, and dark black corresponding to conventional bright field, alumina, and metal oxide crystallites, respectively. Images indicated a uniform and narrow size distribution of dispersed metal oxide crystallites over alumina which confirmed the XRD results. TEM images display uniform size below $10 \mathrm{~nm}$ for metal oxide crystallites on the support. Statistical analysis was carried out to determine the size distribution of metal oxides reported in Table 5 using the Clemex vision software confirming that $\mathrm{La}-\mathrm{Cu} / \mathrm{AlSE}$ catalyst had the smallest crystallites among the bimetallic catalysts.

The nature of surface species and composition of the copper, cobalt, lanthanum and nickel were investigated by XPS measurements. XPS graph of fresh calcined Cu8/AlSE with three sharp peaks is shown in Fig. 11. Comparing the values of the binding energy with those in the open literature, the two main peaks situated at 933.5 and $953.7 \mathrm{eV}$ belong to $\mathrm{Cu}^{2+}$ in $\mathrm{CuO}$. The presence of the satellite peak for $\mathrm{Cu} 2 \mathrm{P}_{3 / 2}$ core level spectra illustrates vaguely the presence of $\mathrm{Cu}^{2+}$ on the surface of support. Furthermore, no satellite peaks were detected in studying $\mathrm{Cu}^{2+}$ and $\mathrm{Cu}^{0}$ which is in a good agreement with previous reports [19-21]. The XPS pattern of La2-Cu6/A1SE is also seen in Fig. 12. The peak at binding energy of $933.4 \mathrm{eV}$ $\left(\mathrm{Cu} 2 \mathrm{P}_{3 / 2}\right)$ indicates that copper exists on La2-Cu6/A1SE Only as a copper oxide $(\mathrm{CuO})$. The binding energy for $\mathrm{La}_{3 / 2}$ level at $835 \mathrm{eV}$ originates from $\mathrm{La}^{3+}$ is present as $\mathrm{La}_{2} \mathrm{O}_{3}$, which could be identified by XRD results [22-23]. Fig.13 shows the XPS of fresh Co2-Cu6/AlSE with the main peak situated at $780 \mathrm{eV}$ belong to $\mathrm{Co}^{2+}$ in $\mathrm{CoO}$. The $\mathrm{Co} 2 \mathrm{P}_{1 / 2}$ binding energy of the catalyst is $795 \mathrm{eV}$, it suggests the presence of $\mathrm{Co}_{2} \mathrm{O}_{3}$ in the surface layer of the alumina as a support [24]. The XPS result of Ni2-Cu6/AlSE is shown in Fig. 14. The binding energy for $\mathrm{Ni} 2 \mathrm{P}_{3 / 2}$ level at $855.8 \mathrm{eV}$ originates from $\mathrm{Ni}^{2+}$ is present as $\mathrm{Ni}_{2} \mathrm{O}_{3}$. The peak at the binding 
energy of $873.8 \mathrm{eV}\left(\mathrm{Ni}_{2} \mathrm{P}_{1 / 2}\right)$ suggests the presence of $\mathrm{NiO}$ on the surface of the catalyst [25]

which can confirm XRD findings.

\section{Catalytic Performance}

\subsection{Screening experiments for selection of the support}

To investigate the effect of support preparation method on catalyst performance in the conversion of toluene in humid air, experiments were carried out over $1 \mathrm{~g}$ of catalyst at a reaction temperature of $350^{\circ} \mathrm{C}$ using a feed with flow rate of $166 \mathrm{ml} / \mathrm{min}$, toluene concentration of $10000 \mathrm{ppmv}$, and relative humidity of $0-94 \%$. Toluene conversions as a function of time-on-stream are presented in Fig.15 for Cu8/AlSE, Cu8/AlCP, and Cu8/AlSM catalysts indicating that after an initial drop in catalyst activity, toluene conversions reached steady values. For all three catalysts toluene conversion decreased with increasing relative humidity from 0 to $94 \%$ during contact time. Fig. 15 also indicates that the maximum conversion of toluene was achieved by Cu8/AlSE for both levels of feed relative humidity. Oxidation products including water vapor and carbon dioxide as well as the water vapor present in the humid feed can cover the hydrophilic active sites of alumina thus reducing the catalyst activity. Results illustrated that toluene conversions were 60.9, 43.7 and 30.2\%over Cu8/AlSE, Cu8/AlCP, and Cu8/AlSM, respectively using a feed with relative humidity of 94\%.The higher catalytic activity for Cu8/AlSE could be due to higher dispersion of smaller size of metal oxides over the $\gamma$-alumina support.

\subsection{Effect of oxidation temperature on the performance of Cu8/AISE}

Fig.16 presents the toluene conversion data obtained for Cu8/A1SE using a feed with relative humidity in the range of $0-94 \%$ and reaction temperatures in the range of $250-400^{\circ} \mathrm{C}$ while keeping other operating conditions the same as reported above. As indicated in Fig.16, toluene conversions were higher for dry feed at all reaction temperatures. Results also 
indicated that after a sharp decrease over the initial stage of the reaction (up to 20 minutes from the start of a run), toluene conversions reached steady values at about 60 min from the start of a run. The catalyst is highly active at the start of a run and as the reaction proceeds, oxidation products such as carbon dioxide compete with toluene to occupy the active metal sites thus lowering the toluene conversions. The highest toluene conversions were 93.6, 91.8, and $82.8 \%$ over $\mathrm{Cu} 8 / \mathrm{AlSE}$ at $400^{\circ} \mathrm{C}$ with relative humidity of 0,40 and $94 \%$, respectively. The present study demonstrated that relative humidity did not have a significant effect on toluene conversions at either low reaction temperatures $\left(250^{\circ} \mathrm{C}\right)$ where the catalyst activity is very low or at high reaction temperatures $\left(400^{\circ} \mathrm{C}\right)$ where the catalyst activity is high and the competitive adsorption of water vapor is less likely.

\subsection{Catalytic performance over bimetallic catalysts}

Fig. 17 presents toluene conversions over La-Cu/AlSE, Co-Cu/AlSE, and Ni-Cu/AlSE bimetallic catalysts at reaction temperature of $350^{\circ} \mathrm{C}$ and relative humidity of $94 \%$ while keeping all other operating conditions the same as previously stated. Fig.17a indicates that addition of lanthanum and copper oxides to the alumina support reduces the catalyst activity for complete oxidation of toluene despite the surface modification caused by simultaneous addition of lanthanum and copper. Activity of copper oxide for total oxidation of toluene is higher than other metal oxides as reported in the literature [4]. Compared with other La$\mathrm{Cu} / \mathrm{A} 1 \mathrm{SE}$ bimetallic catalysts, therefore, La2-Cu6/A1SE had the highest activity for oxidation of toluene in the humid air. Fig.17b and Fig. 17c also show a similar trend of decreasing catalyst activity with increasing cobalt content of $\mathrm{Co}-\mathrm{Cu} / \mathrm{AlSE}$ catalysts and with increasing nickel content of Ni-Cu/AlSE catalysts, respectively. Maximum toluene conversions of 90.2, 76.4, and 64\% were obtained over La2-Cu6/AlSE, Co2-Cu6/A1SE, and Ni2-Cu6/A1SE, respectively. Fig.18 shows the long term performance of La2-Cu6/AlSE for toluene 
conversion for a feed with relative humidity of $94 \%$ indicating that after the initial decrease, catalyst activity remained constant for up to 54 hours.

Toluene might be oxidized over supported metal oxides catalysts at temperatures below those for thermal combustion in the presence of water vapor. Supported noble and transition metal oxides are widely applied for complete oxidation of toluene. Li et al. [3] found that the conversion of toluene in the presence of water vapor was near $100 \%$ at $330^{\circ} \mathrm{C}$ with an inlet feed toluene concentration of $1200 \mathrm{ppmv}$ over $\mathrm{Cu}-\mathrm{Mn} \mathrm{O}_{\mathrm{x}} / \mathrm{gamma}-\mathrm{Al}_{2} \mathrm{O}_{3}$ in the comparison with the supported catalysts over $\mathrm{TiO}_{2}$ and cordierite. They used commercial alumina for synthesize nano catalysts. Other copper based catalysts supported on commercial alumina, cord and cordierite was also studied for total oxidation of toluene in the presence water vapor, Jiancai et al. [26], who reported a high activity of copper oxide supported on gamma-alumina for reaction at $450^{\circ} \mathrm{C}$ and inlet feed concentration of $3.5 \mathrm{gm}^{-3}$. Synthesized bimetallic catalysts in this work have been utilized at $350^{\circ} \mathrm{C}$ as an oxidation temperature.

Plasma catalysis system and photocatalytic oxidation are another approaches for complete oxidation of toluene in the humidified air which has been investigated in many researches [27-28]. Wu et al. [27] found that supported nickel oxide catalysts over gammaalumina, SBA-15 and $\mathrm{TiO}_{2}$ can be appropriate catalysts for oxidation of toluene. Nickel oxide catalyst over commercial alumina had a good performance for complete oxidation of toluene in comparison with other supports. To the best of our knowledge, using nano gamma-alumina prepared by sol-gel method for synthesis of dispersed supported nano bimetallic catalysts for oxidation of toluene is addressed for the first time in our investigation.

\section{Conclusion}

Monometallic and bimetallic catalysts were prepared using $\gamma$-alumina as a support for total oxidation of toluene in humid air at atmospheric pressure. Three series of $\gamma$-alumina were 
synthesized by the sol-gel method with either ethanol or methanol as a solvent and by the coprecipitation method. Catalytic activity of supported copper oxide on the $\gamma$-alumina synthesized by the sol-gel method with ethanol as a solvent (AlSE) was higher compared with the same catalyst supported on other $\gamma$-alumina supports. Heterogeneous depositionprecipitation method was used for preparation of bimetallic catalysts supported on AlSE with different ratios of copper oxide to oxide of other metals including lanthanum, cobalt, and nickel. The type and ratio of the second metal had significant effects on the size of the metal oxide crystallites, the formation of different crystallite phases, the dispersion of active sites, the surface area and the morphology of the prepared catalyst samples, and the catalyst activity. Although the addition of the second metal led to enhanced dispersion of copper and better surface characteristics, the activity of supported bimetallic catalysts ( $\mathrm{La}, \mathrm{Co}$, or $\mathrm{Ni}$ )-Cu/AlSE decreased with increasing lanthanum, cobalt, or nickel loading on the $\gamma$-alumina. Among the three series of bimetallic catalysts, those prepared with copper and lanthanum resulted in the best performance for complete oxidation of toluene in air. Antonym effect of water vapor in reactor feed was investigated which had no significant effect on the catalyst activity at either low reaction temperatures $\left(250^{\circ} \mathrm{C}\right)$ where the catalyst activity is very low or at high reaction temperatures $\left(400^{\circ} \mathrm{C}\right)$ where the catalyst activity is high and the competitive adsorption of water vapor is less likely.

\section{Acknowledgement}

The authors gratefully acknowledge the financial assistance of Iran National Science Foundation (INSF).

\section{References}

[1] Zabihi, M., Khorasheh, F., Shayegan, J., RSC Adv. 5(2015) 5107. 
[2] Mo, J., Zhang, Y., Xu, Q., Applied Catalysis B: Environmental,132-133(2013) 212218.

[3] Li, X., Wang, L., Xia, Q., Liu, Z., Li, Z. Catalysis Communications, 14 (2011) 15-19.

[4] Zabihi, M., Shayegan, J., Fahimirad, M., Khorasheh, F., Journal of Porous Materials, 22 (2015) 101-118.

[5] Einaga, H., Futamura, S., Journal of Catalysis, 243 (2006) 446-450.

[6] Kircher, O., Hougen, O.A., AlChE Journal, 3 (1957) 331-335.

[7] Arnold, E. W., Sundaresan, S., Applied Catalysis 41 (1988) 225-239.

[8] Saqer, S.M., Kondarides, D.I., Verykios, X.E., Applied Catalysis B: Environmental 103 (2011) 275-286.

[9] Kim, K.,Boo1, S., Ahn, H., Journal of Industrial and Engineering Chemistry, 15(2009) 92-97.

[10] Santillan-Jimenez, E., Miljković-Kocić, V.,Crocker, M.,Wilson, K., Applied Catalysis B: Environmental, 102(2011) 1-8.

[11] Carrillo, A.M., Carriazo, J.G., Applied Catalysis B: Environmental,443-452 (2015) 164-170.

[12] Lee, D.S., Chen, Y.W., Journal of the Taiwan Institute of Chemical Engineers,4 (2013) 40-44.

[13] Ebadi, A., Safari, N., Applied Catalysis A,321(2011) 135-13.

[14] Xu, L., He, C.,Catalysis Letters,114(2011) 1-4.

[15] Rogojan, R., Andronescu, E.,Ghitulica, C., Vasile, B.S., U.P.B. Science Bulletin, Series B,73 (2011) 1454.

[16] Xu, L., Song, H., Chou, L., Materials Chemistry and Physics, 132 (2012)1071-1076.

[17] Rajaeiyan, A., Bagheri-Mohagheghi, M.M., Advances in Manufacturing, 1 (2013) $176-181$. 
[18] Parida, K.M., Pradhan, A.C., Das, J., Sahu, N., Materials Chemistry and Physics, 113 (2009) 244-248.

[19] Lo’ pez-Sua'rez F.E., Bueno-Lo’ pez A., Illa’n-Go’ mez M.J., Applied Catalysis B: Environmental, 84 (2008) 651-658.

[20] Gervasini A., Manzoli M., Martra G., Ponti A., Ravasio N., Sordelli L., Zaccheria F., J. Phys. Chem. B 110 (2006) 7851-7861.

[21] Vila F., López Granados M., Ojeda M., Fierro J.L.G., Mariscal R., Catalysis Today 187 (2012) 122-128.

[22] Xirong C., Zaoming C., Weiming L., Advanced Materials Research Vols 347-353 (2012) 3616-3620.

[23] Yanga R., Zhang Z., Wu J., Li X., Wang L., Kinetics and Catalysis 56 (2015) 222225.

[24] Zhang Y., Zhang Y., Feng C., Qiu C., Wen Y., Zhao J., Catalysis Communications, 10 (2009) 1454-1458.

[25] Yun Y.S , Park D.S., Yi J., Catal. Sci. Technol. 4 (2014) 3191-3197.

[26] Jiancai F., Xiao C., Qibin X., Hongxia X., Zhong L., Chinese J. Chem. Eng. 17 (2009) 767-772.

[27] Wu J., Xia Q., Wang H., Li Z., Appl. Catal. B: Environ. 156-157 (2014) 265-272. 
Table 1. Crystallite size of alumina prepared by the sol-gel and co-precipitation methods

\begin{tabular}{lcc}
\hline Sample & Crystallite phase & Crystallite size (nm) \\
\hline \multirow{2}{*}{ AlCP } & Large & 6.3 \\
& Small & 3.5 \\
\multirow{2}{*}{ AlSE } & Large & 4.7 \\
& Small & 2.3 \\
\hline
\end{tabular}

Table 2.Textural properties of the prepared $\gamma$-Alumina by different methods

\begin{tabular}{llll}
\hline$\gamma$-Alumina & $S_{B E T} / \mathrm{m}^{2} \cdot \mathrm{g}^{-1}$ & $\mathrm{~V} / \mathrm{cm}^{3} \cdot \mathrm{g}^{-1}$ & $\mathrm{D} / \mathrm{nm}$ \\
\hline AlCP & 305 & 0.2988 & 5.653 \\
AlSE & 284 & 0.3734 & 8.771 \\
AlSM & 97 & 0.1351 & 9.215 \\
\hline
\end{tabular}

Table 3. Crystallite size of the prepared catalysts over $\gamma$-alumina byheterogeneous depositionprecipitation method

\begin{tabular}{lcc}
\hline Sample & Crystallite phase & Crystallite size (nm) \\
\hline \multirow{2}{*}{ Cu8/AlCP } & Large & 8.2 \\
& Small & 3.5 \\
\multirow{2}{*}{ Cu8/AlSE } & Large & 6.1 \\
& Small & 3.3 \\
\multirow{2}{*}{ Cu8/A1SM } & Large & 20.4 \\
& Small & 8.4 \\
\hline
\end{tabular}

Table 4. Textural properties of the prepared catalysts over $\gamma$-alumina by heterogeneous deposition-precipitation method

\begin{tabular}{llll}
\hline Catalyst & $S_{B E T} / \mathrm{m}^{2} \cdot \mathrm{g}^{-1}$ & $\mathrm{~V} / \mathrm{cm}^{3} \cdot \mathrm{g}^{-1}$ & $\mathrm{D} / \mathrm{nm}$ \\
\hline Cu8/AlCP & 204 & 0.3107 & 6.0927 \\
Cu8/AlSE & 154 & 0.4121 & 10.682 \\
Cu8/AlSM & 53 & 0.1417 & 10.955 \\
\hline
\end{tabular}


Table 5. Crystallite size of the bimetallic catalysts over $\gamma$-alumina

\begin{tabular}{|c|c|c|}
\hline Sample & Crystallite phase & Crystallite size (nm) \\
\hline \multirow{2}{*}{ La2-Cu6/A1SE } & Large & 6.1 \\
\hline & Small & 2.9 \\
\hline \multirow{2}{*}{ La4-Cu4/A1SE } & Large & 5.8 \\
\hline & Small & 2.8 \\
\hline \multirow{2}{*}{ La6-Cu2/A1SE } & Large & 4.6 \\
\hline & Small & 2.6 \\
\hline \multirow{2}{*}{ Co2-Cu6/A1SE } & Large & 4.1 \\
\hline & Small & 2.9 \\
\hline Co4-Cu4/AlSE & Large & 5.2 \\
\hline \multirow{3}{*}{$\mathrm{Co6}-\mathrm{Cu} 2 / \mathrm{AlSE}$} & Small & 2.8 \\
\hline & Large & 8.9 \\
\hline & Small & 3.5 \\
\hline \multirow{2}{*}{ Ni2-Cu6/AlSE } & Large & 7.9 \\
\hline & Small & 2.8 \\
\hline \multirow{2}{*}{ Ni4-Cu4/AlSE } & Large & 10.7 \\
\hline & Small & 2.9 \\
\hline \multirow{2}{*}{ Ni6-Cu2/AlSE } & Large & 9 \\
\hline & Small & 5.3 \\
\hline
\end{tabular}


Fig. 1.Schematic diagram of the experimental set-up

Fig. 2.XRD patterns of AlCP and AlSE

Fig. 3.FESEM images for AlCP and AlSE

Fig. 4.Nitrogen adsorption-desorption results for AlCP, AlSE, and AlSM

Fig. 5.XRD patterns of Cu8/AlCP, Cu8/AlSE, and Cu8/AlSM

Fig. 6.FESEM images for Cu8/AlCP, Cu8/AlSE, and Cu8/AlSM

Fig. 7.TEM images for Cu8/A1SE

Fig. 8.Nitrogen adsorption-desorption results for Cu8/AlCP, Cu8/A1SE, and Cu8/AlSM

Fig. 9. a)XRD patterns for the prepared bimetallic catalysts over $\gamma$-alumina by heterogeneous deposition-precipitation method $\mathrm{Cu}-\mathrm{La}$

b)XRD patterns for the prepared bimetallic catalysts over $\gamma$-alumina by heterogeneous deposition-precipitation method $\mathrm{Cu}-\mathrm{Co}$

c)XRD patterns for the prepared bimetallic catalysts over $\gamma$-alumina by heterogeneous deposition-precipitation method $\mathrm{Cu}-\mathrm{Ni}$

Fig. 10. a)TEM images for La2-Cu6/A1SE

b) TEM images for Co2-Cu6/A1SE

c)TEM images for Ni2-Cu6/AlSE

Fig. 11.XPS spectra of the Cu8/AlSE

Fig. 12.XPS spectra of the La2-Cu6/A1SE

Fig. 13.XPS spectra of the Co2-Cu6/A1SE

Fig. 14.XPS spectra of the Ni2-Cu6/AlSE 
Fig. 15.Total oxidation of toluene over supported copper catalysts Cu8/AlSE, Cu8/AlCP and $\mathrm{Cu} 8 / \mathrm{AlSM}$ with 0 and $94 \%$ relative humidity of the feed

Fig. 16. a)Total oxidation of toluene over Cu8/AlSE at feed relative humidity of 0 - $94 \%$ and reaction temperatures of $250^{\circ} \mathrm{C}$

b)Total oxidation of toluene over Cu8/AlSE at feed relative humidity of $0-94 \%$ and reaction temperatures of $300^{\circ} \mathrm{C}$

c)Total oxidation of toluene over $\mathrm{Cu} 8 / \mathrm{AlSE}$ at feed relative humidity of $0-94 \%$ and reaction temperatures of $350^{\circ} \mathrm{C}$

d)Total oxidation of toluene over Cu8/AlSE at feed relative humidity of $0-94 \%$ and reaction temperatures of $400^{\circ} \mathrm{C}$

Fig. 17. a)Total oxidation of toluene over the prepared catalysts at feed relative humidity of $94 \%$ and reaction temperatures of $350^{\circ} \mathrm{C}$ for La2-Cu6/A1SE, La4-Cu4/A1SE, La6-Cu2/A1SE, and Cu8/AlSE

b)Total oxidation of toluene over the prepared catalysts at feed relative humidity of $94 \%$ and reaction temperatures of $350^{\circ} \mathrm{C}$ for $\mathrm{Co} 2-\mathrm{Cu} 6 / \mathrm{AlSE}, \mathrm{Co} 4-\mathrm{Cu} 4 / \mathrm{AlSE}, \mathrm{Co} 6-\mathrm{Cu} 2 / \mathrm{AlSE}$, and $\mathrm{Cu} 8 / \mathrm{AlSE}$

c)Total oxidation of toluene over the prepared catalysts at feed relative humidity of $94 \%$ and reaction temperatures of $350^{\circ} \mathrm{C}$ for $\mathrm{Ni2}-\mathrm{Cu} 6 / \mathrm{A} 1 \mathrm{SE}$, Ni4-Cu4/A1SE, Ni6-Cu2/A1SE, and Cu8/AlSE

Fig. 18.Long term performance of La2-Cu6/A1SE for oxidation of toluene at feed relative humidity of $94 \%$ and reaction temperature of $350^{\circ} \mathrm{C}$ 


\section{Figure 1.}

Schematic diagram of the experimental set-up 







\section{Figure 2.}

XRD patterns of AlCP and AlSE 


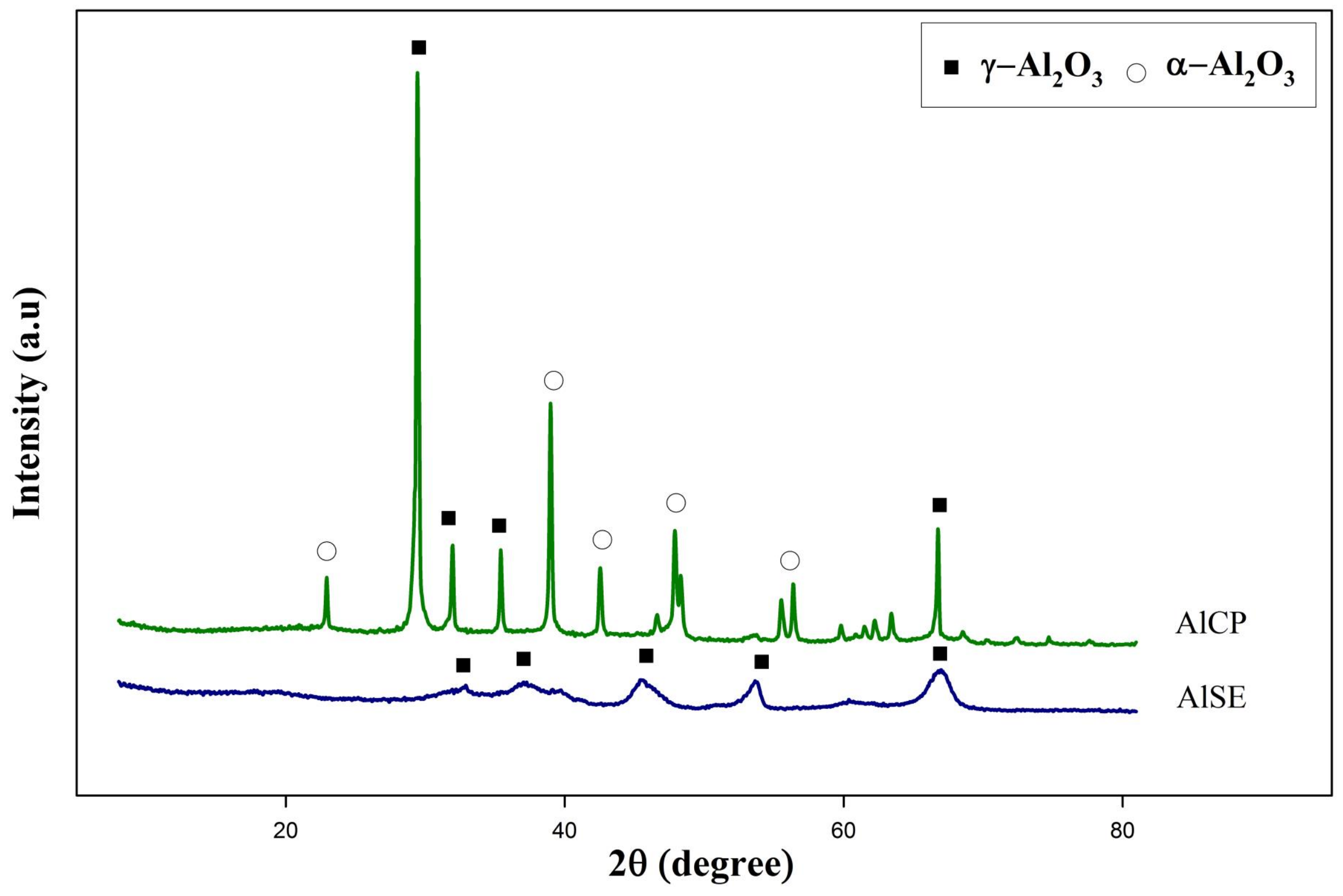




\section{Figure 3.}

FESEM images for AlCP and AlSE 

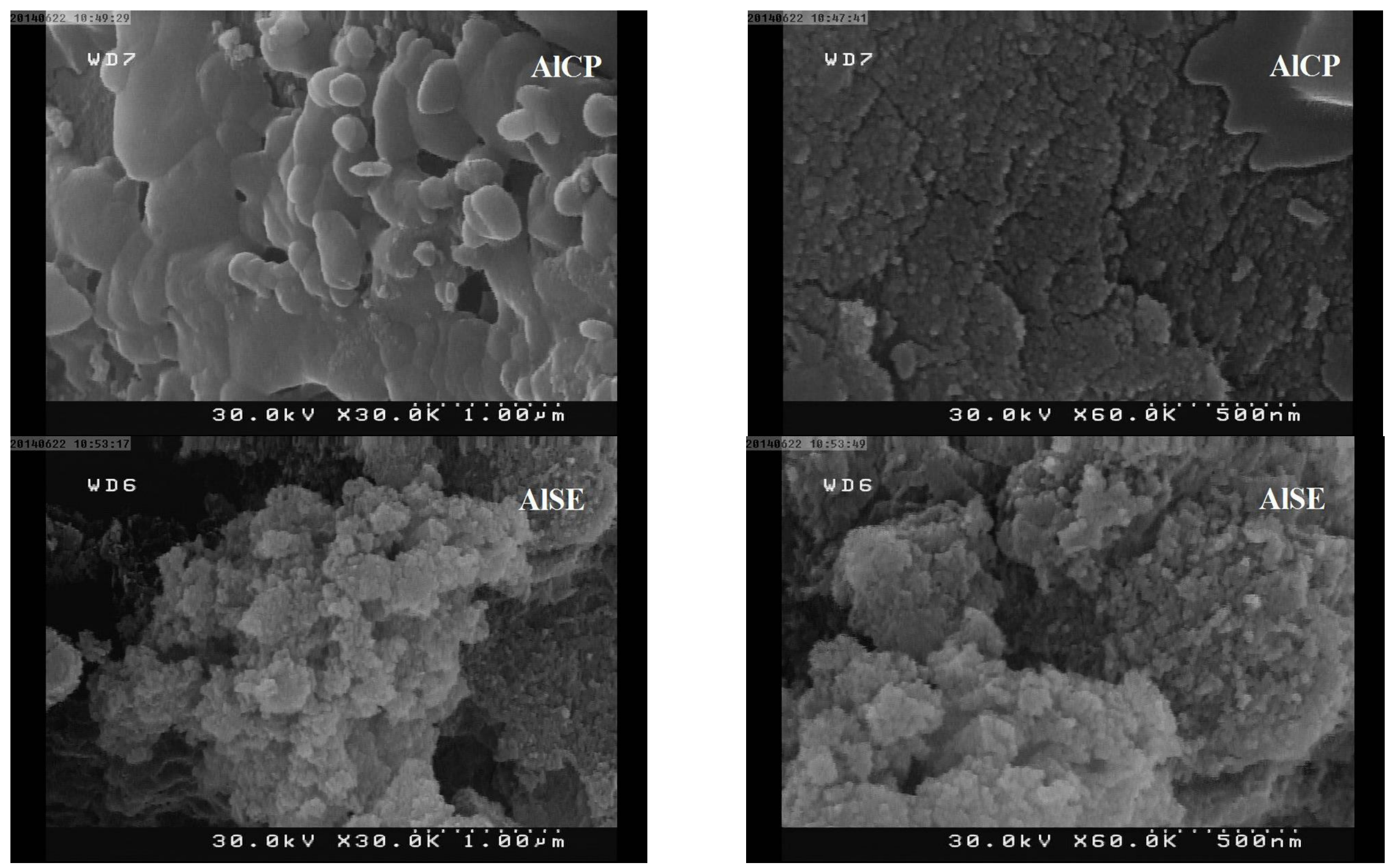


\section{Figure 4.}

Nitrogen adsorption-desorption results for AlCP, AlSE, and AlSM 


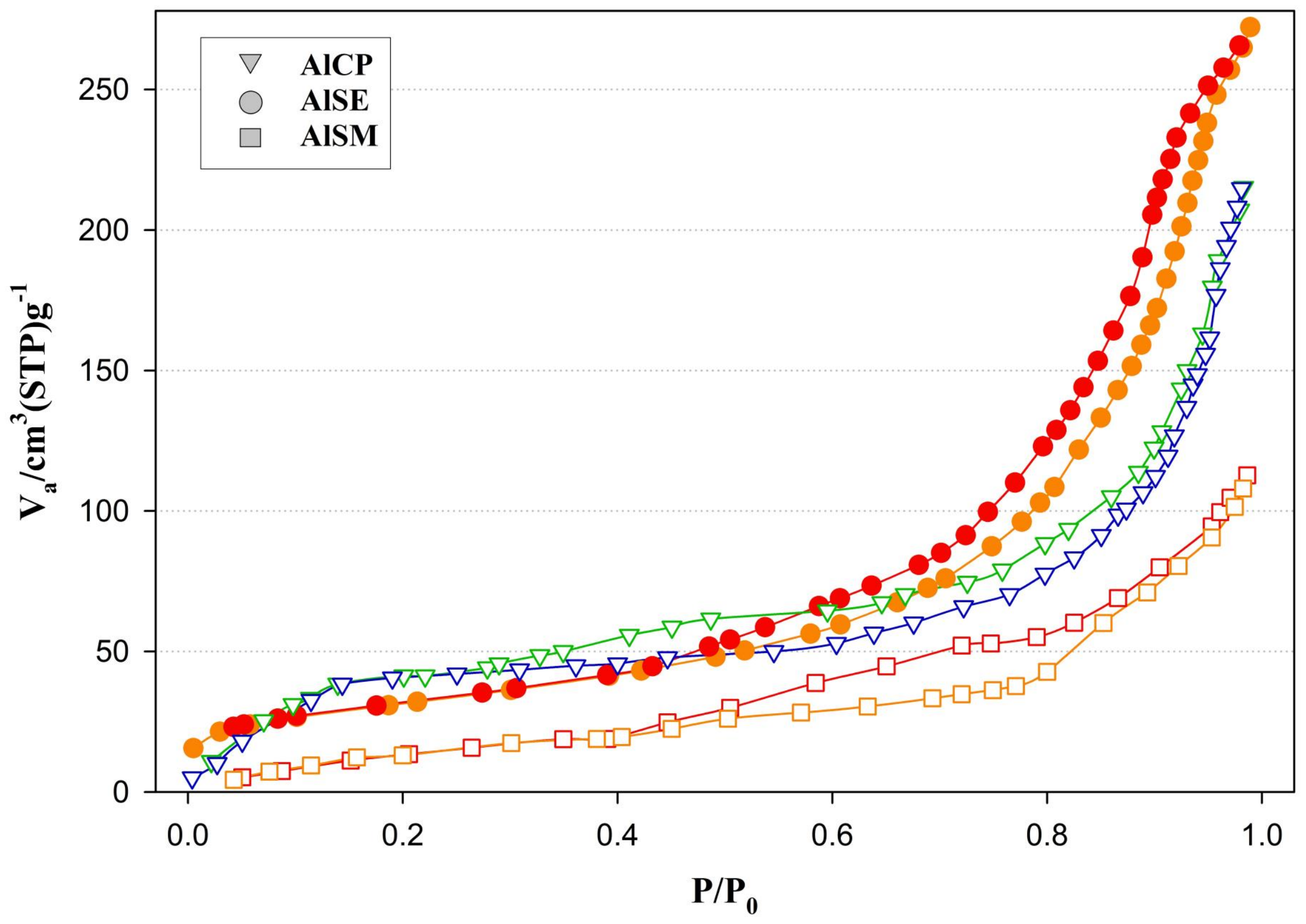




\section{Figure 5.}

XRD patterns of Cu8/AlCP, Cu8/AlSE, and Cu8/AlSM 


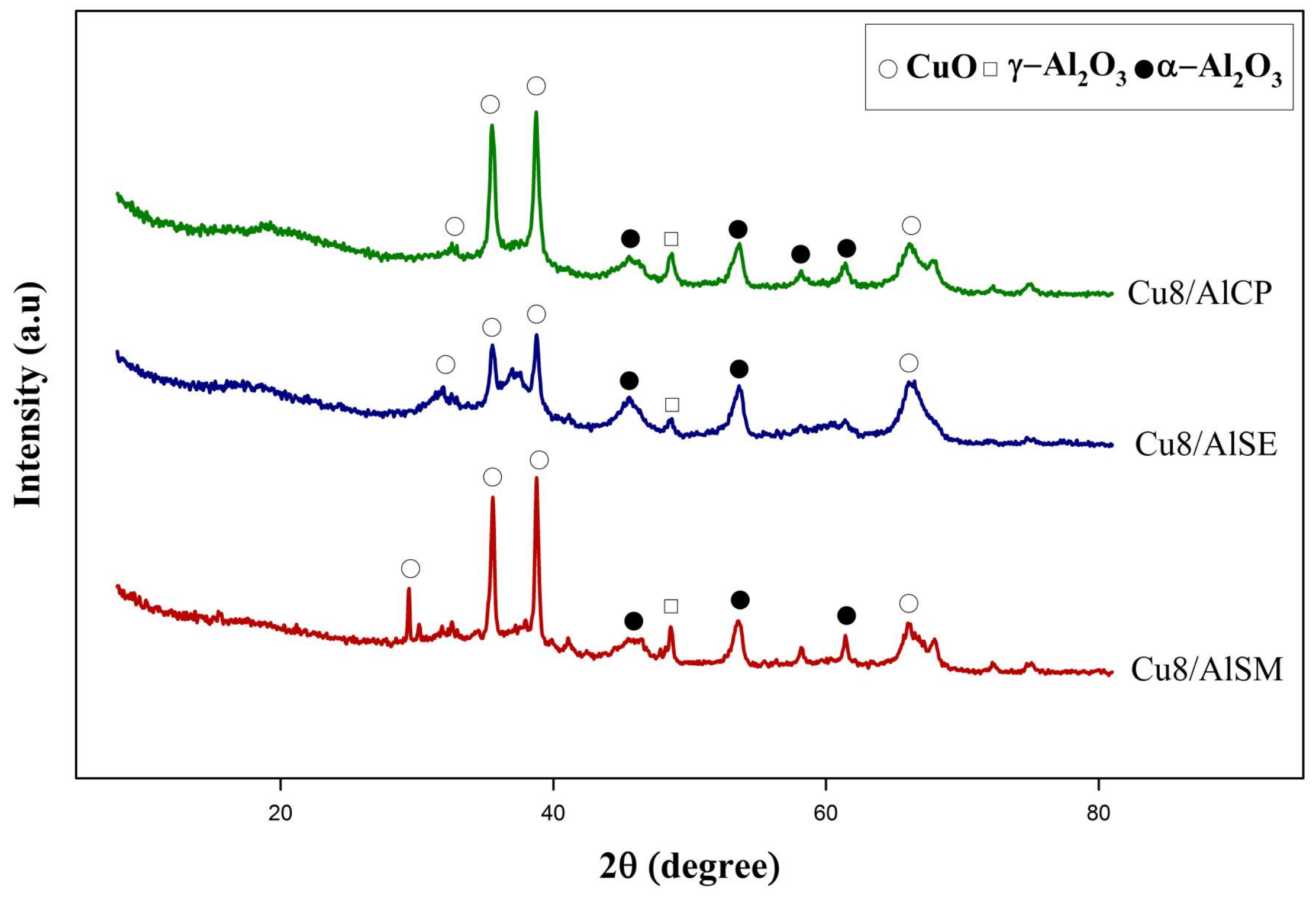




\section{Figure 6.}

FESEM images for Cu8/AlCP, Cu8/AlSE, and Cu8/AlSM 

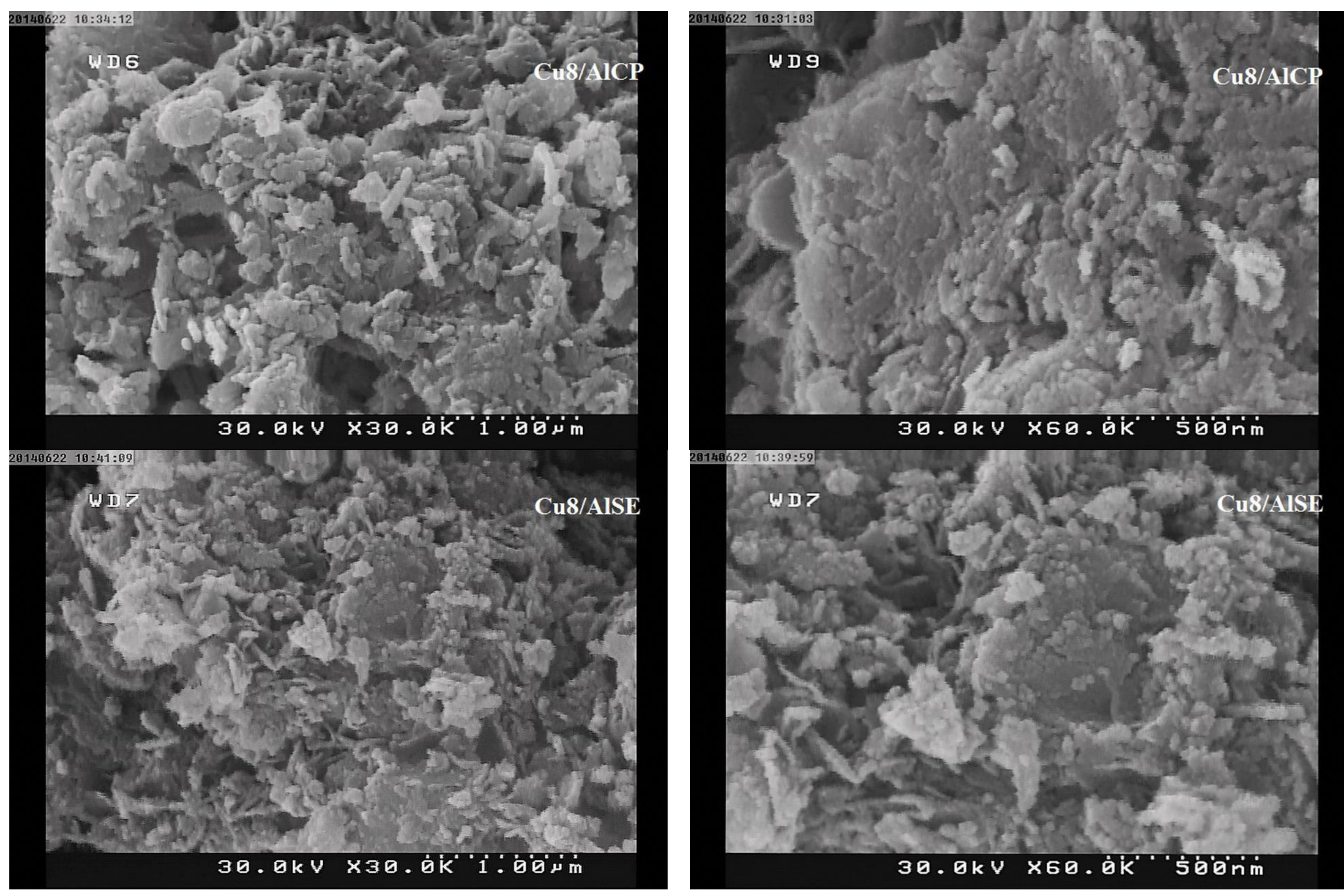

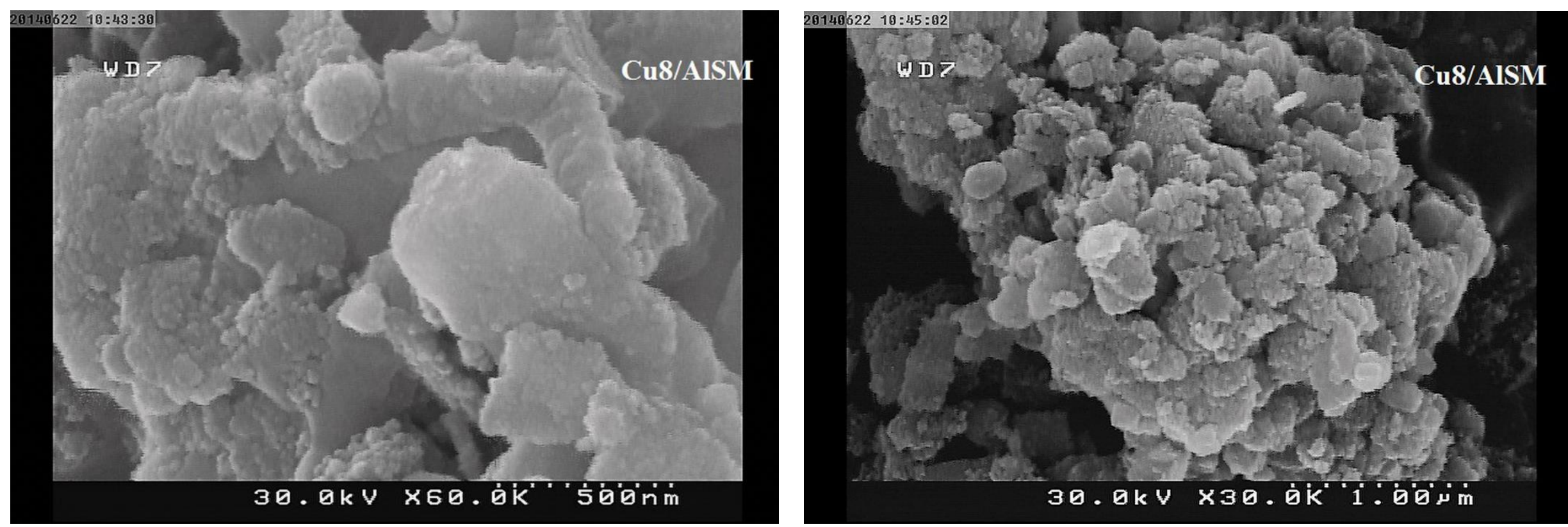


\section{Figure 7.}

TEM images for Cu8/AlSE 

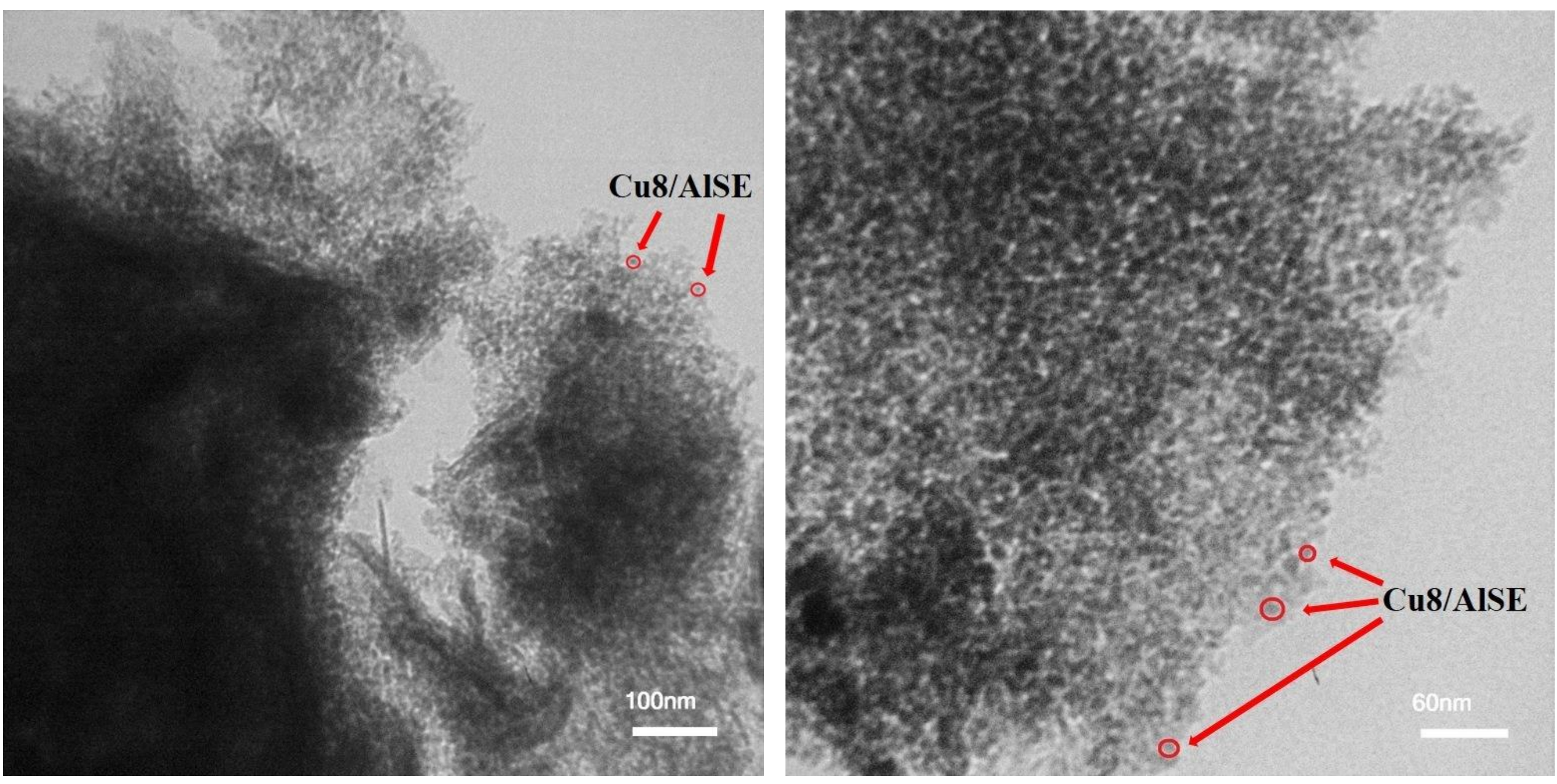


\section{Figure 8.}

Nitrogen adsorption-desorption results for Cu8/AlCP, Cu8/AlSE, and Cu8/AlSM 


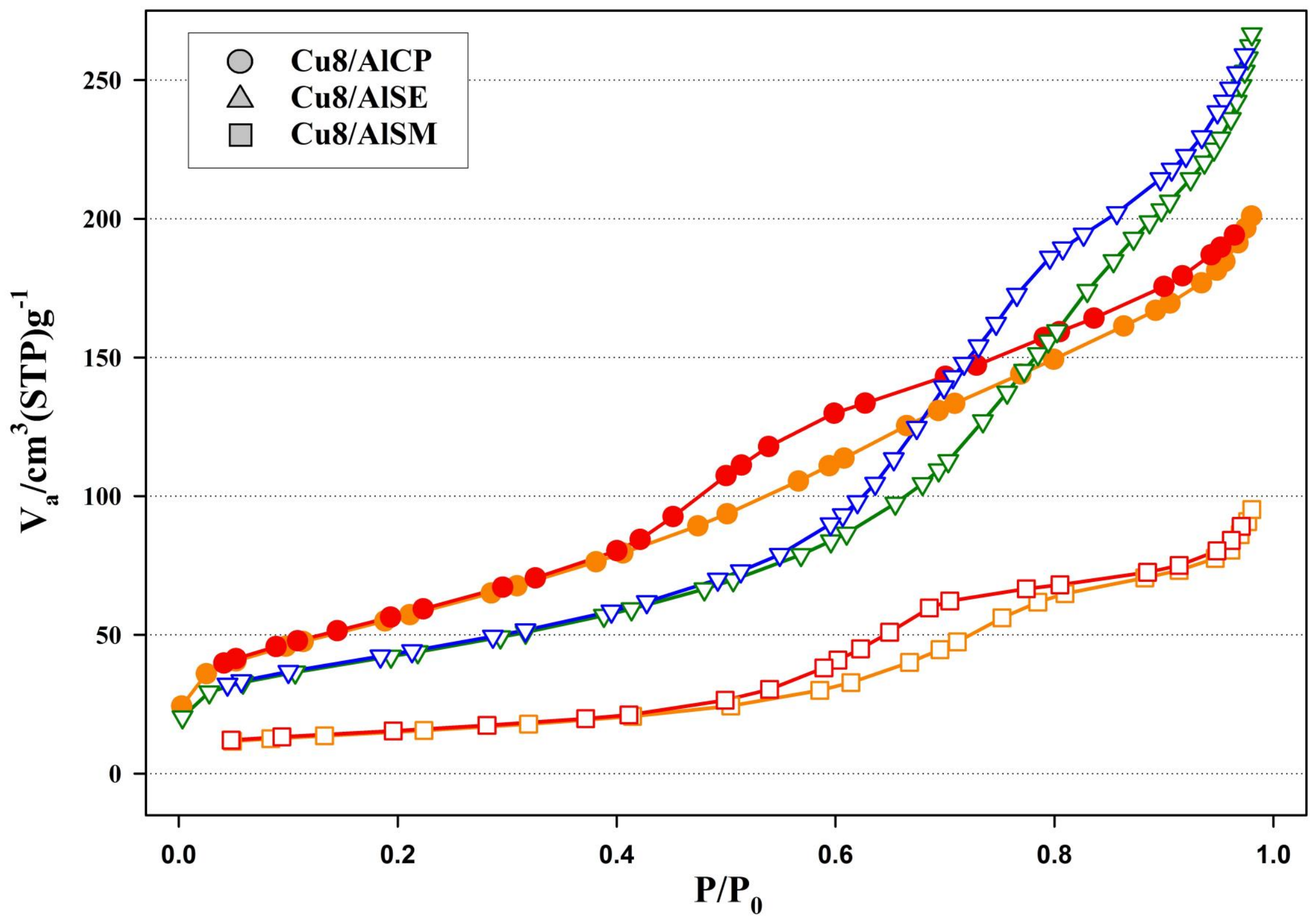




\section{Figure 9.}

XRD patterns for the prepared bimetallic catalysts over $\gamma$-alumina by heterogeneous deposition-precipitation method a) $\mathrm{Cu}-\mathrm{La}$, b) $\mathrm{Cu}-\mathrm{Co}$, and c) $\mathrm{Cu}-\mathrm{Ni}$ 
a)

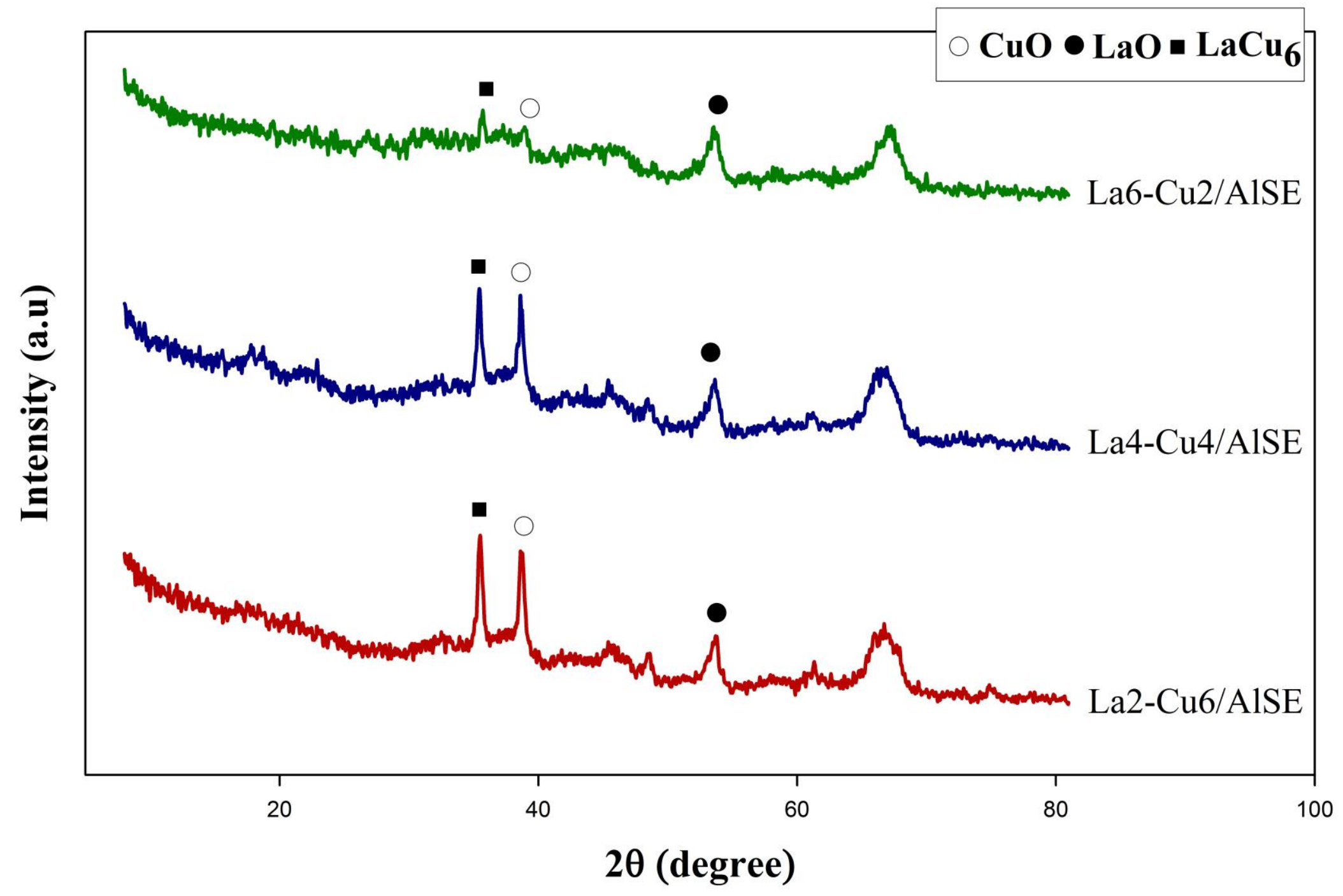


b)

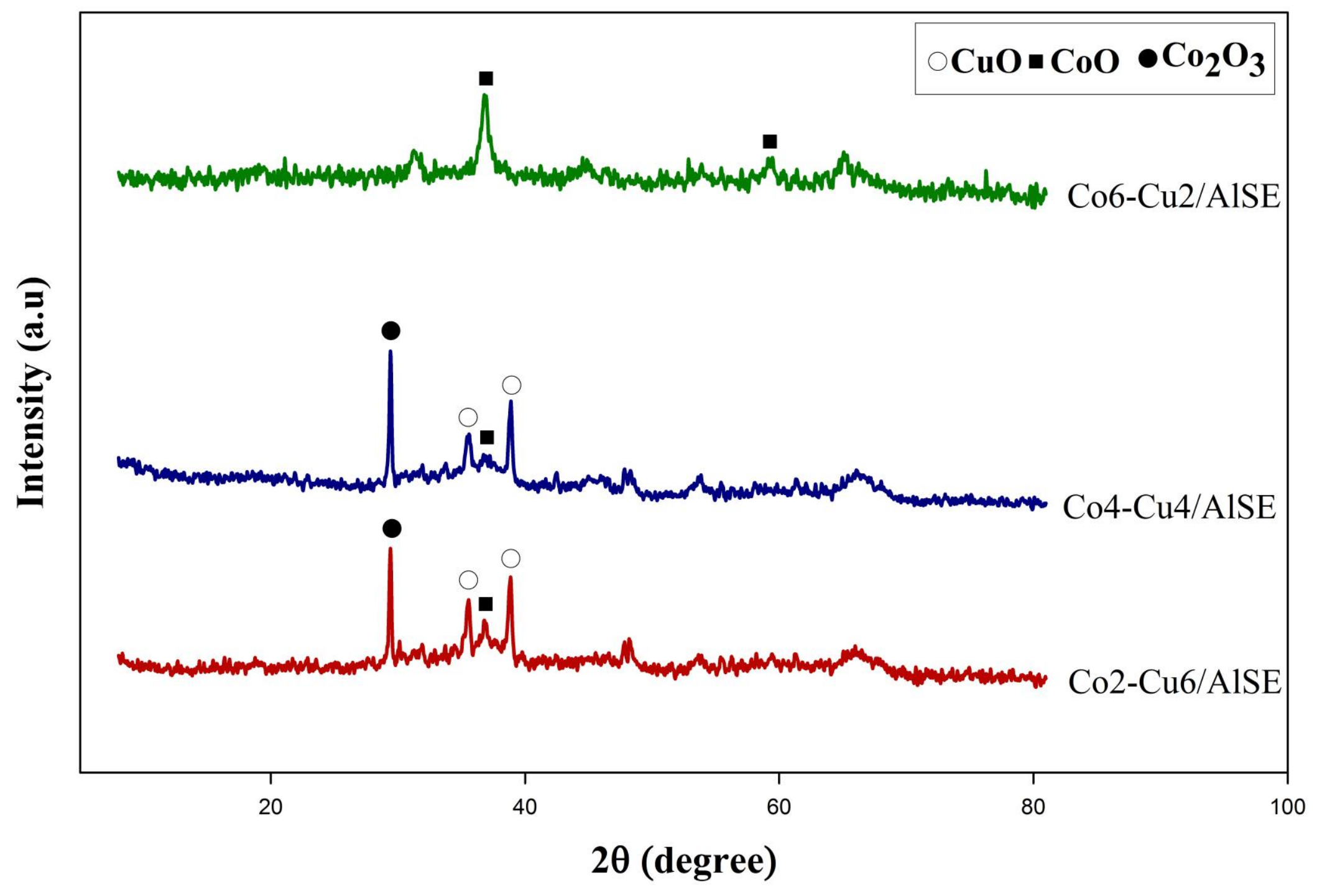


c)

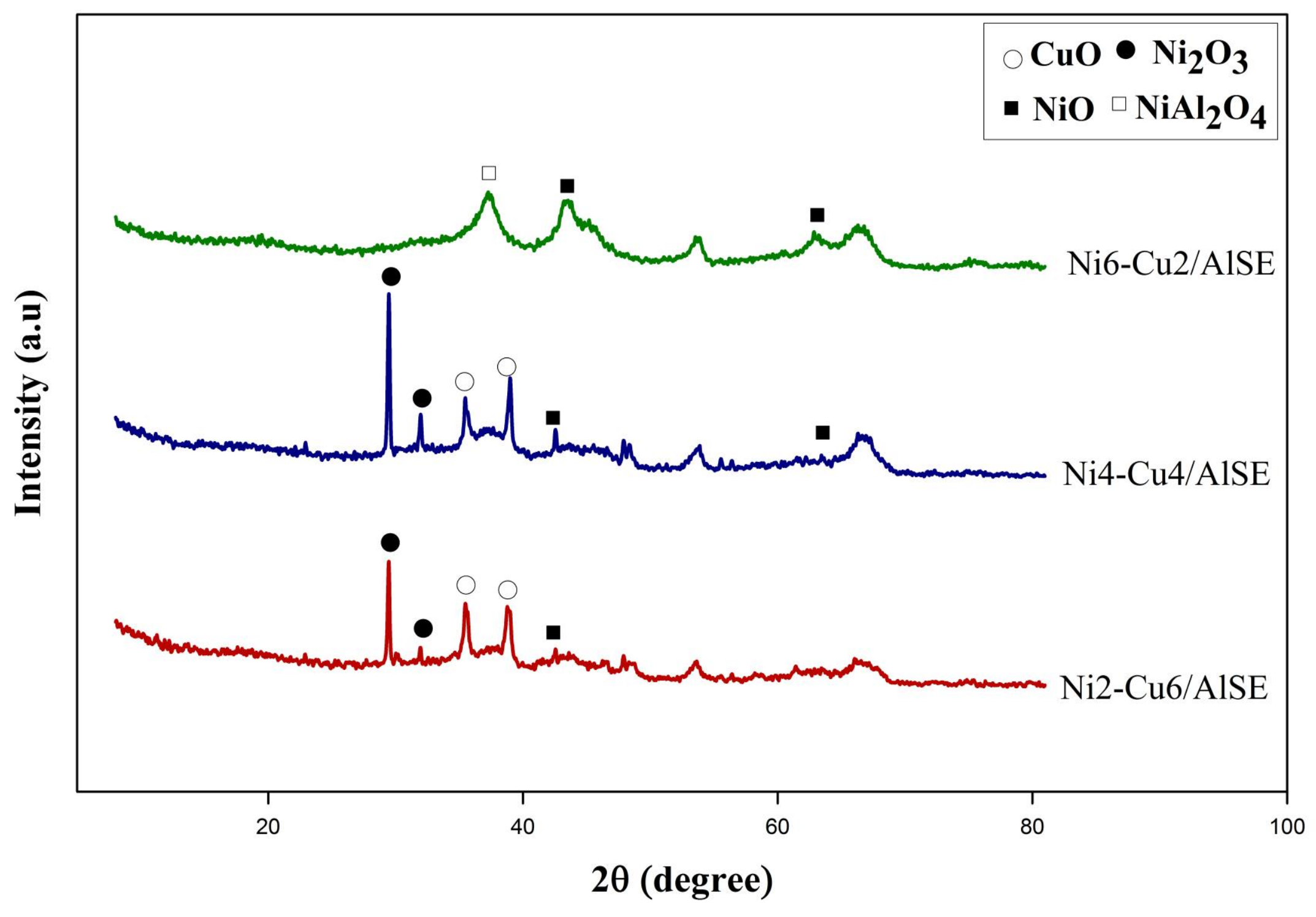




\section{Figure 10.}

TEM images for a) La2-Cu6/AlSE, b) Co2-Cu6/A1SE, and c) Ni2-Cu6/AlSE 


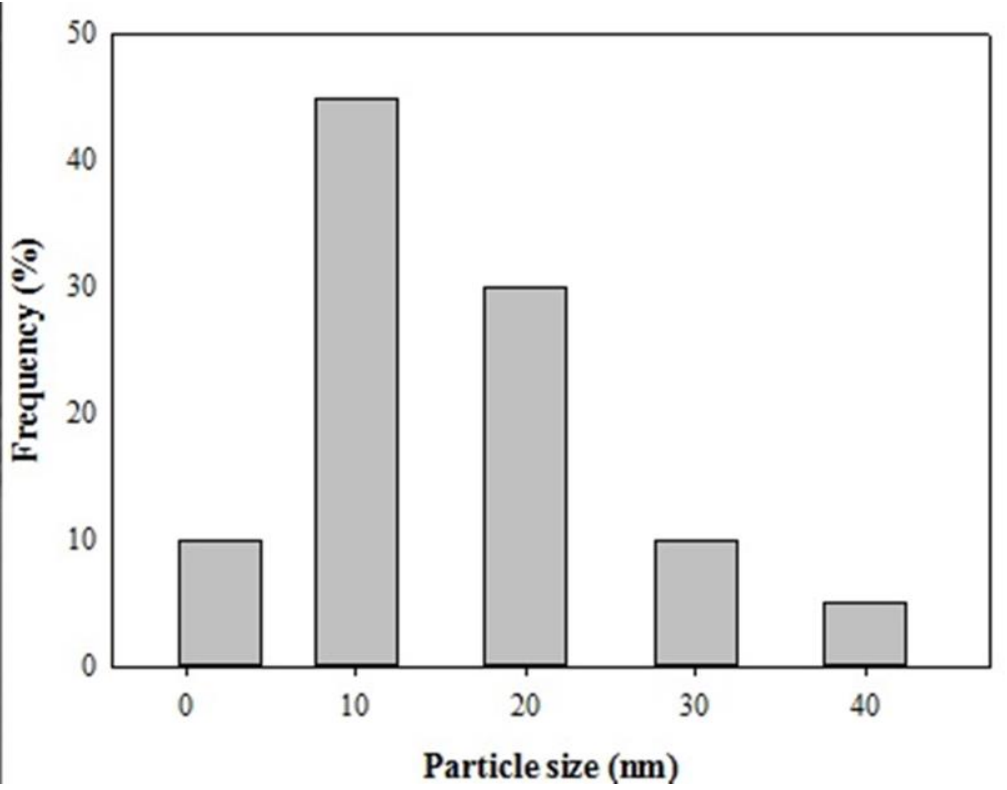


b)

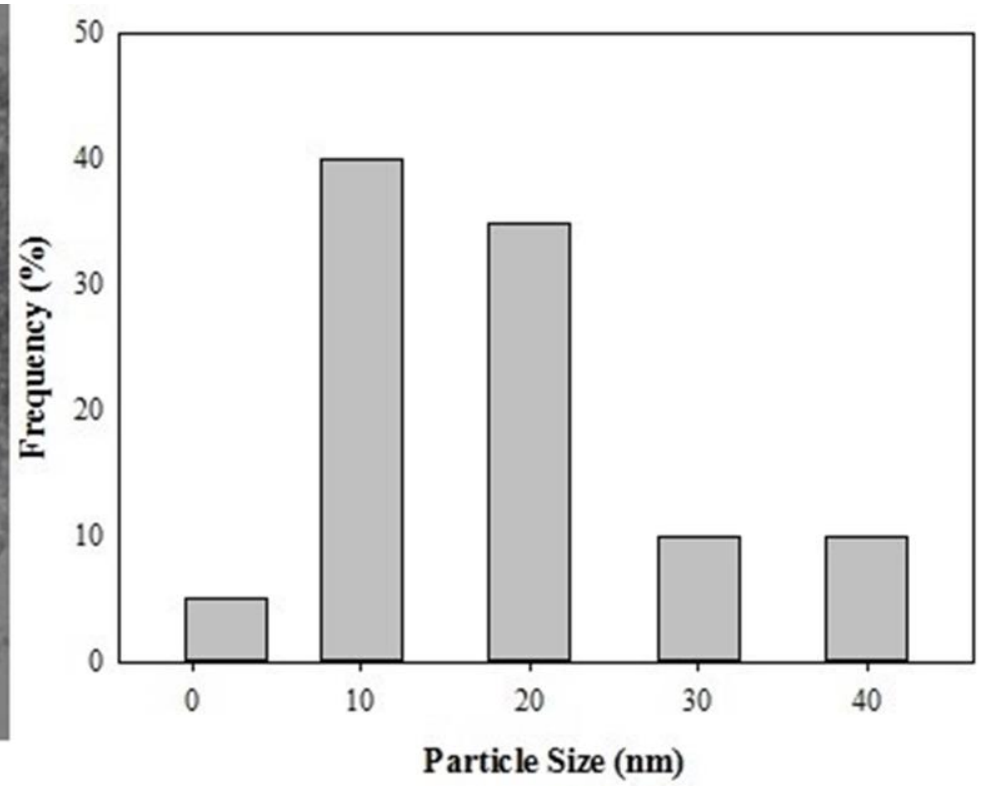



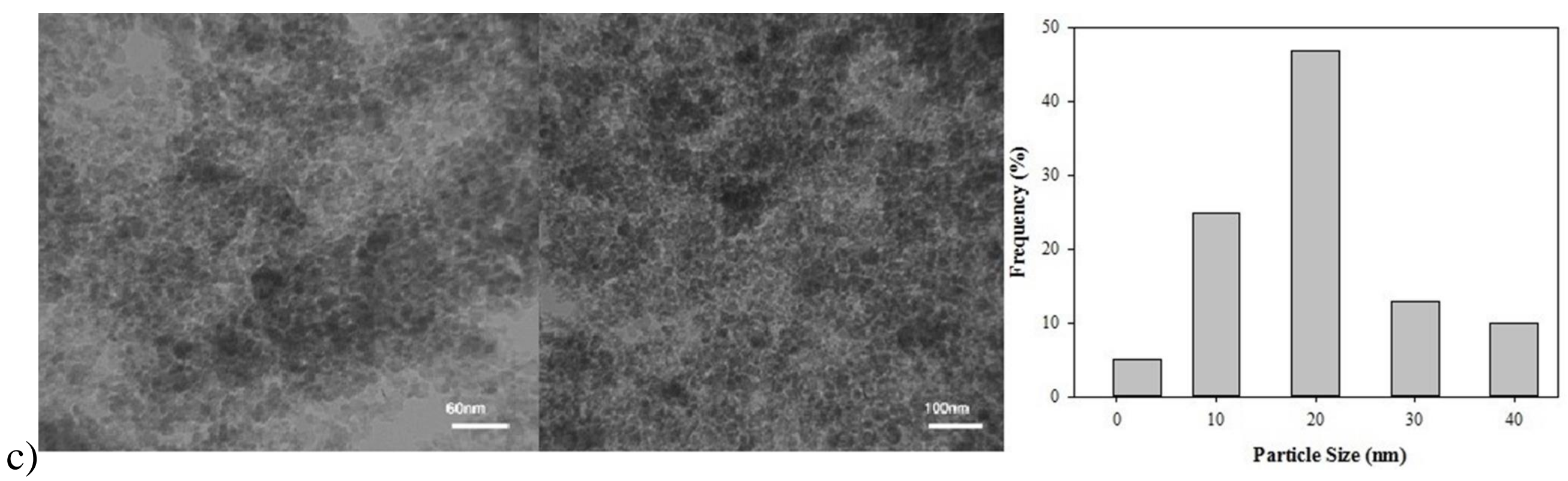


\section{Figure 11.}

XPS spectra of the Cu8/AlSE 


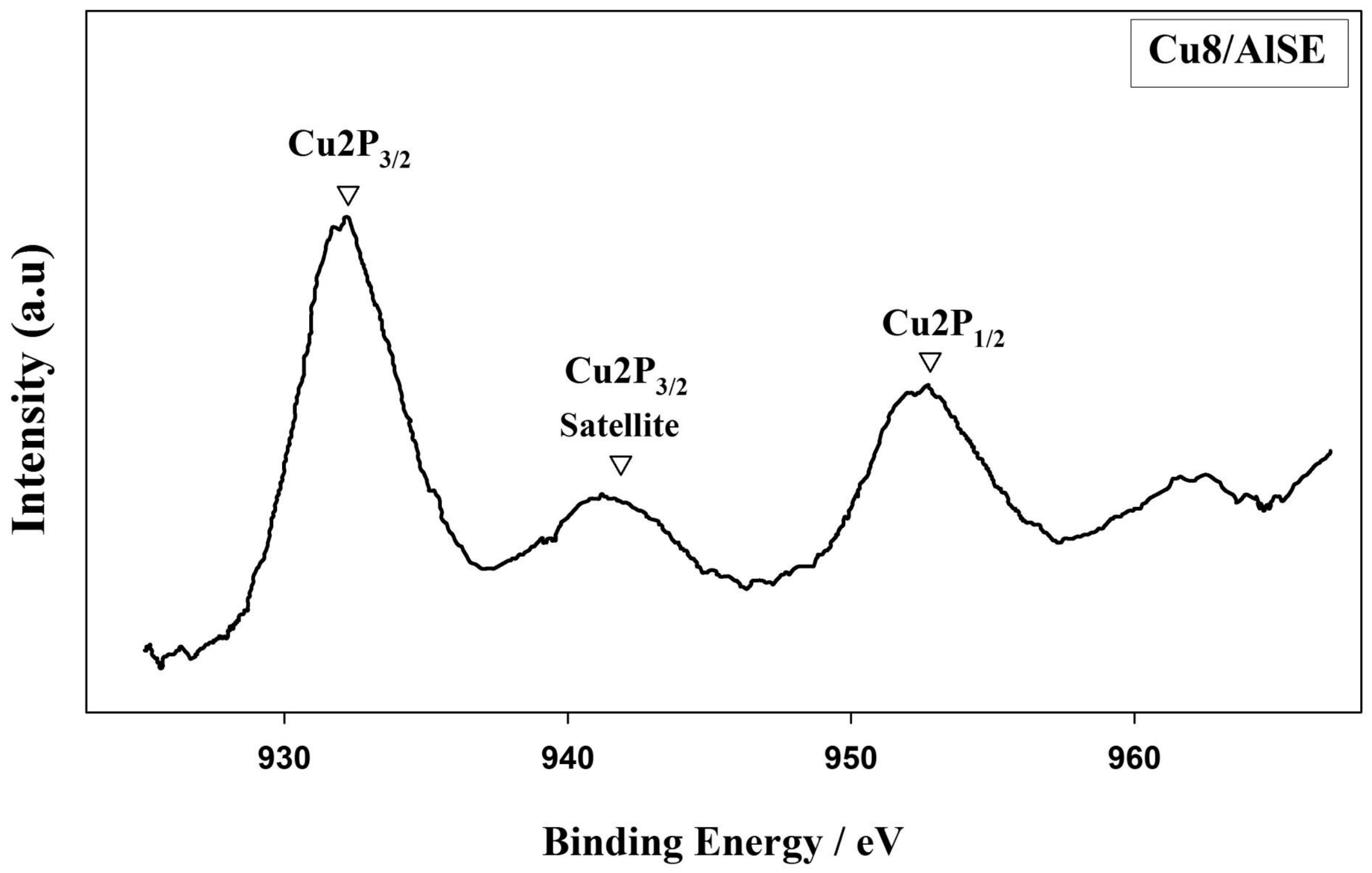




\section{Figure 12.}

XPS spectra of the La2-Cu6/AlSE 


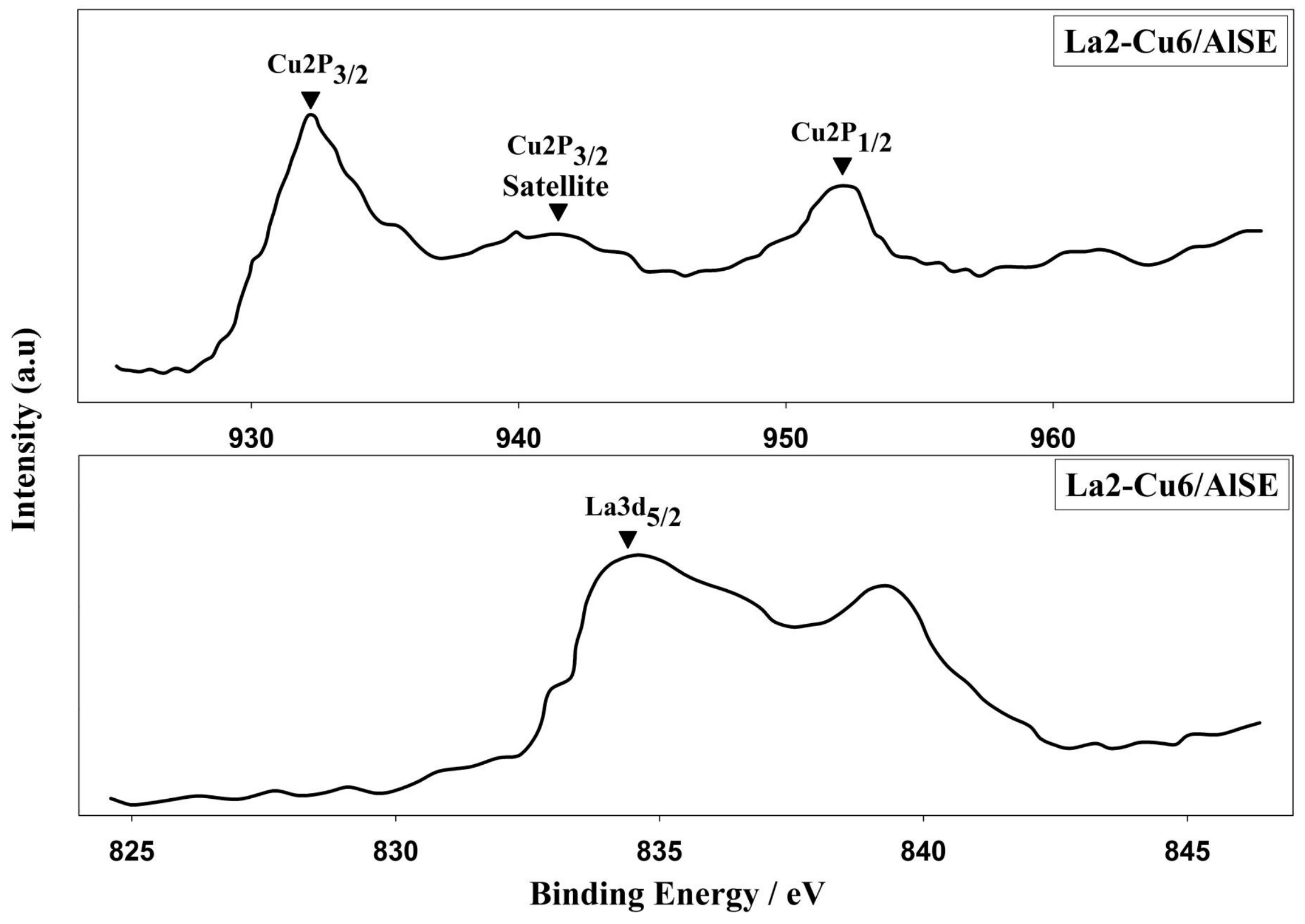




\section{Figure 13.}

XPS spectra of the Co2-Cu6/AlSE 


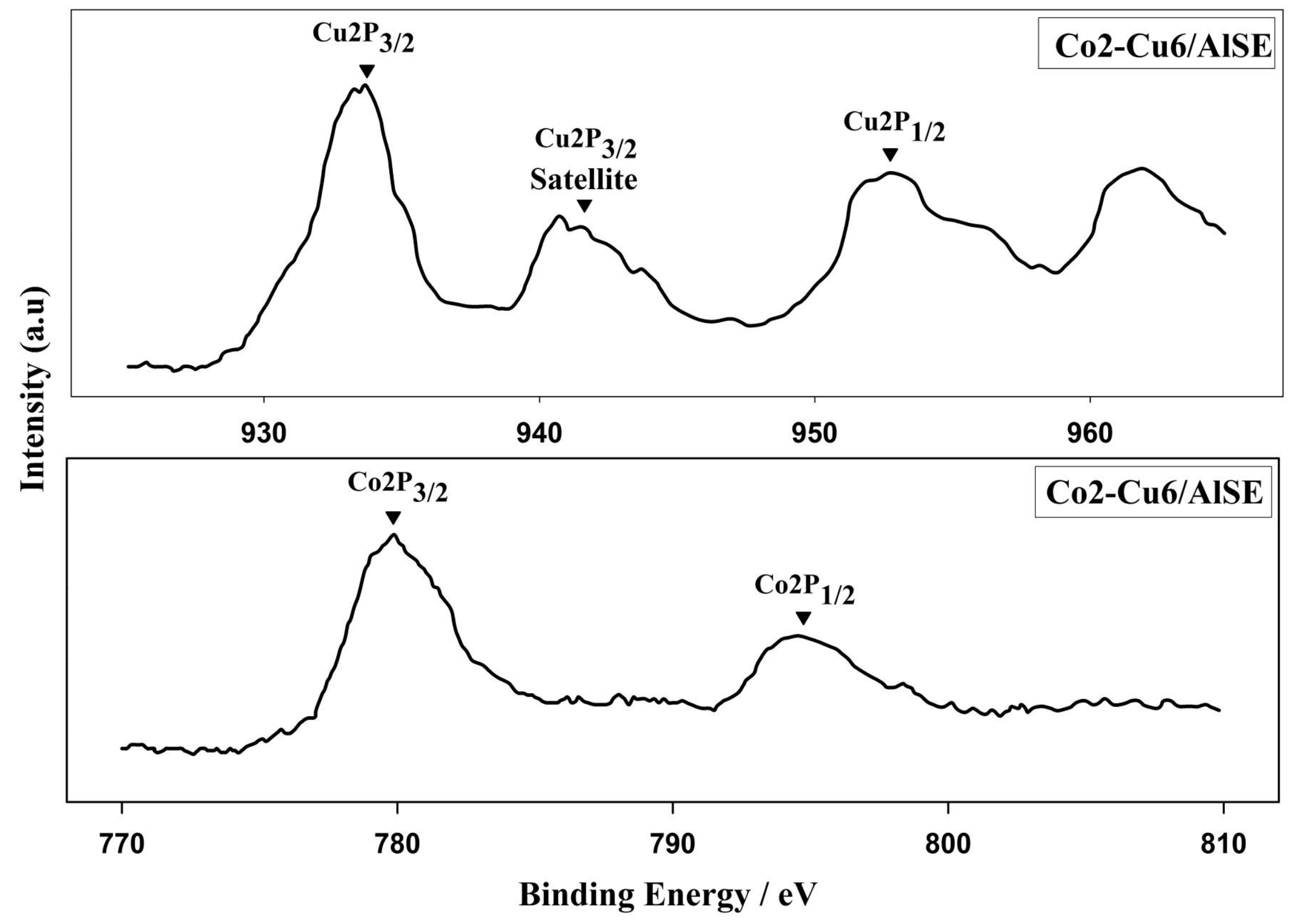




\section{Figure 14.}

XPS spectra of the Ni2-Cu6/AlSE 


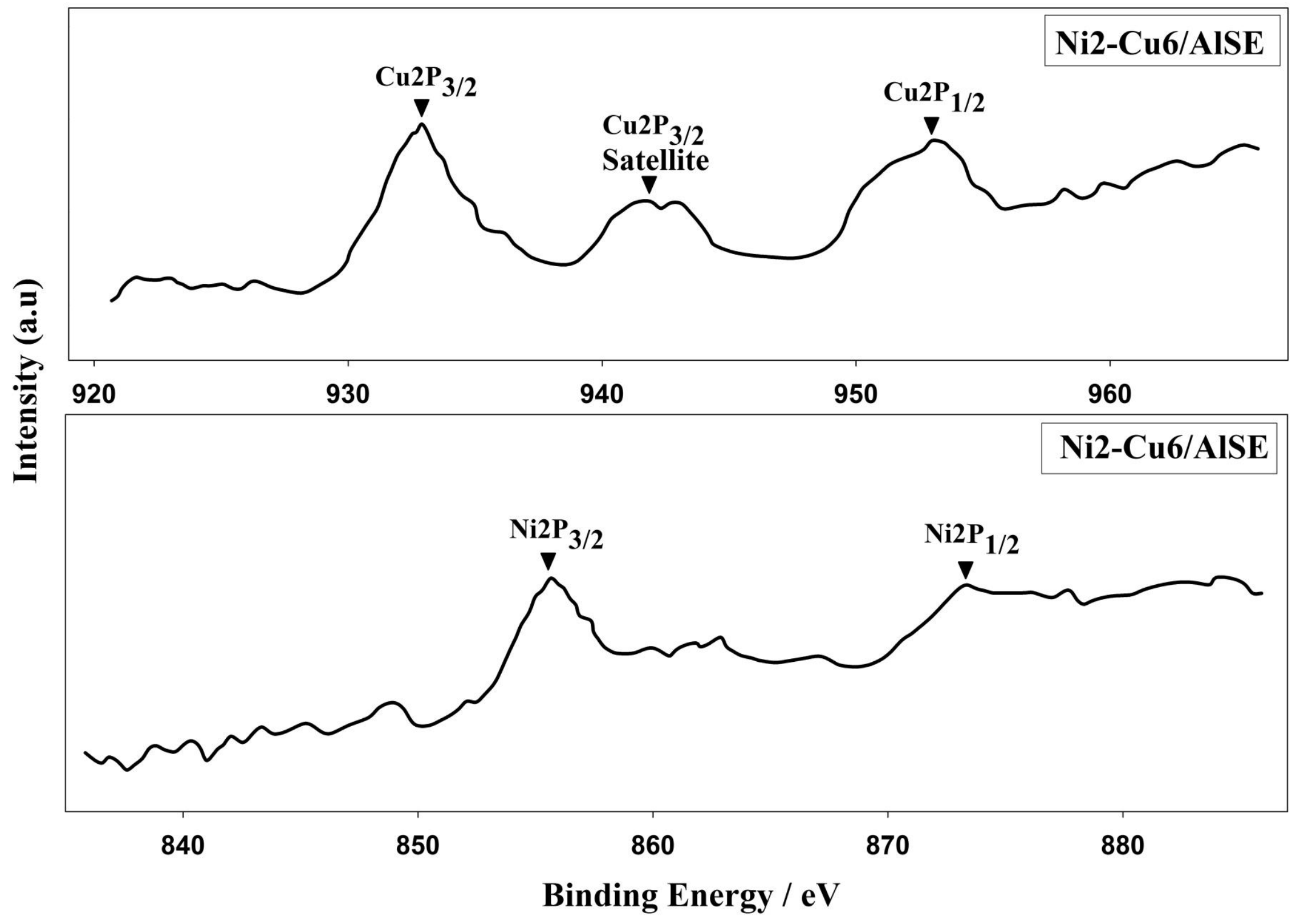




\section{Figure 15.}

Total oxidation of toluene over supported copper catalysts Cu8/AlSE, Cu8/AlCP and Cu8/AlSM with 0 and $94 \%$ relative humidity of the feed 


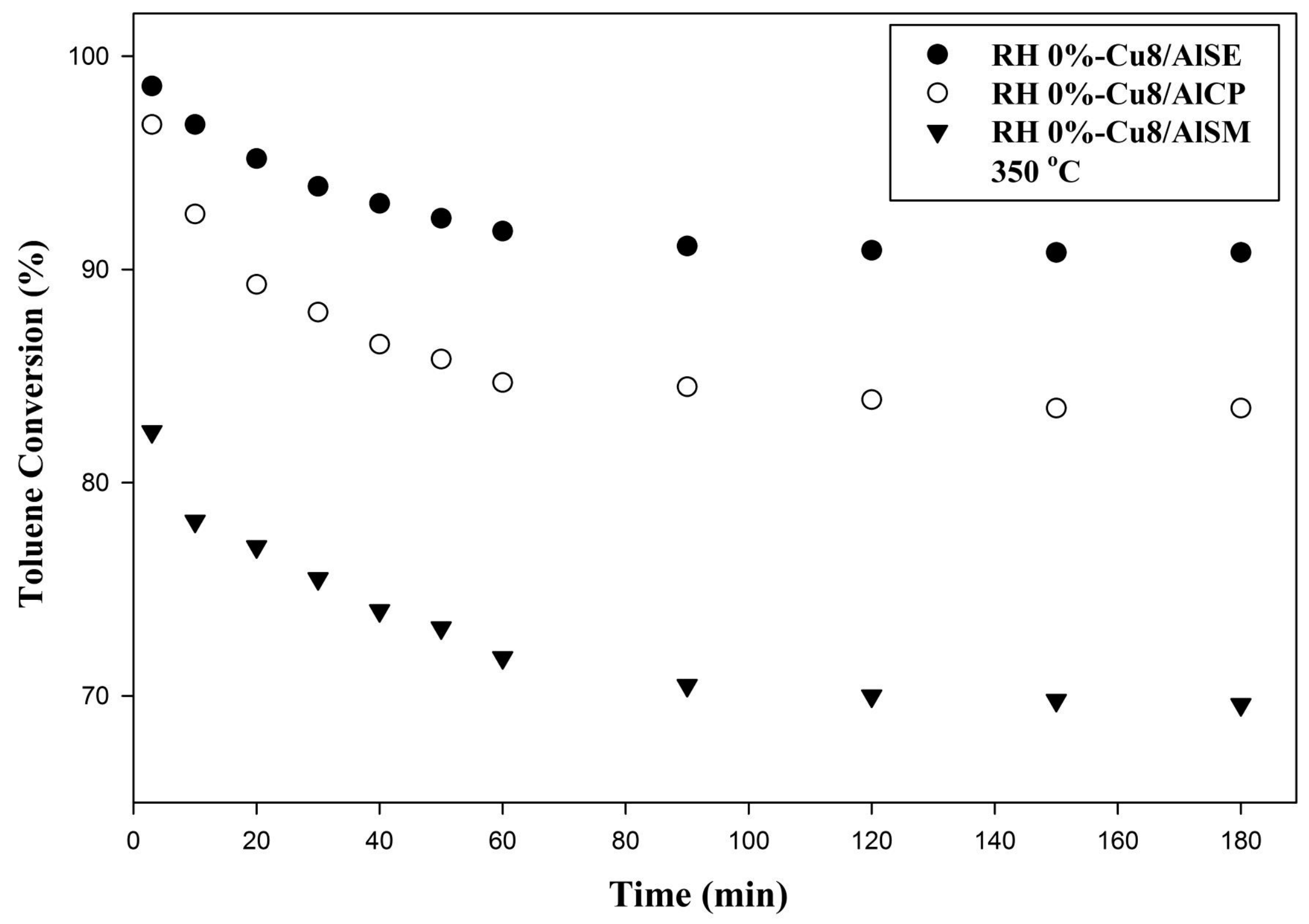




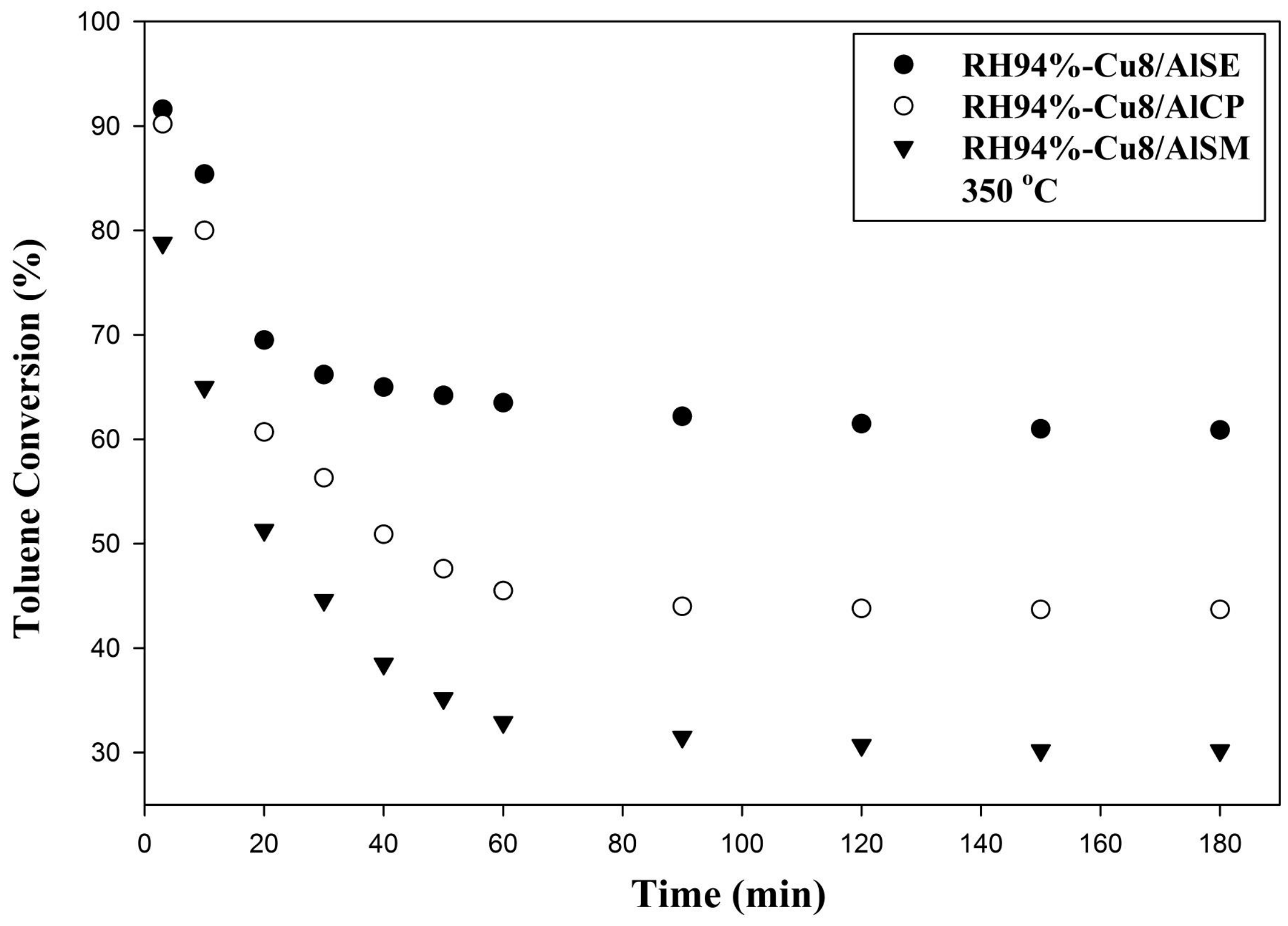




\section{Figure 16.}

Total oxidation of toluene over Cu8/AlSE at feed relative humidity of $0-94 \%$ and reaction temperatures of a) $250^{\circ} \mathrm{C}$, b) $300^{\circ} \mathrm{C}$, c) $350^{\circ} \mathrm{C}$, and d) $400^{\circ} \mathrm{C}$ 


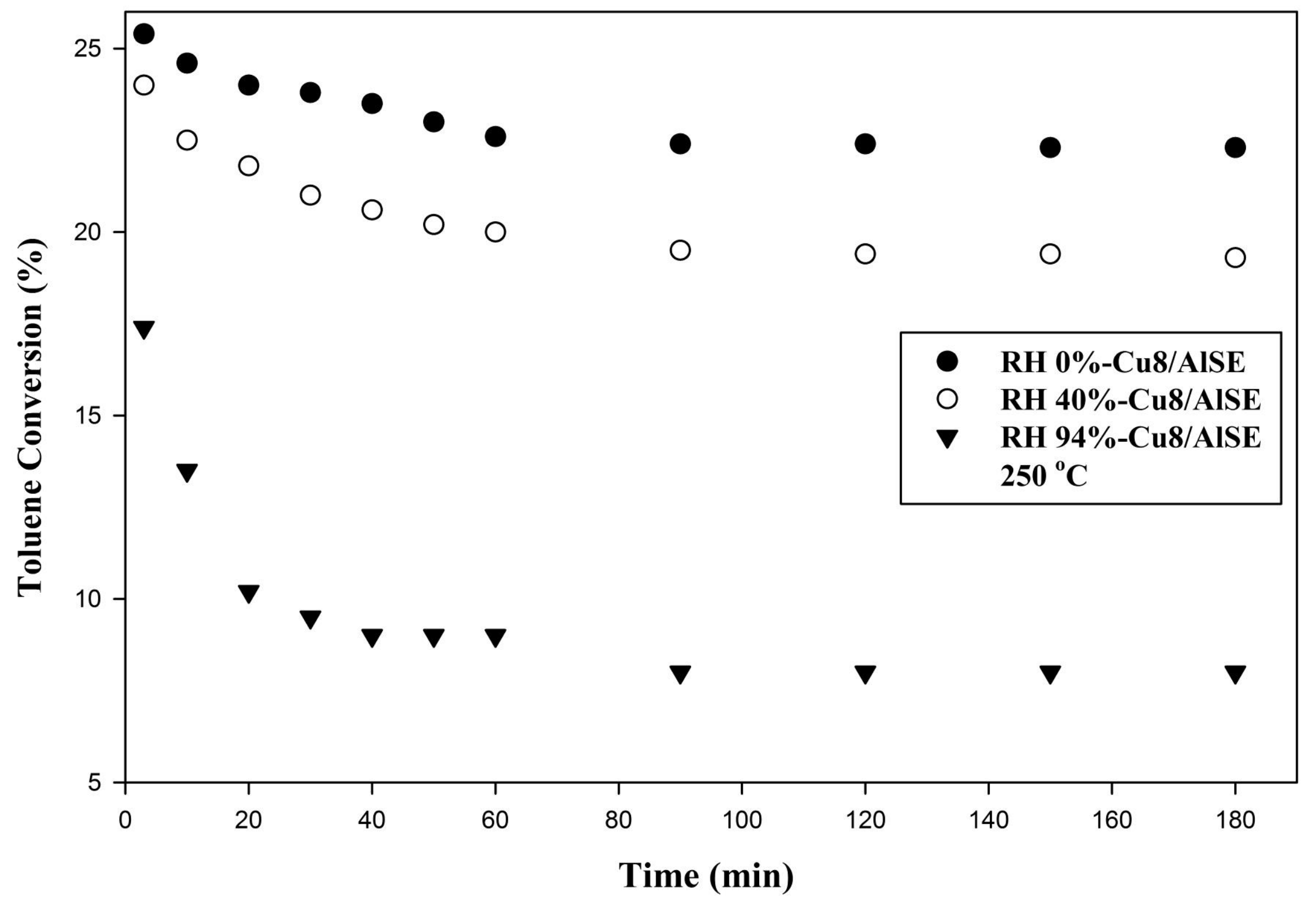




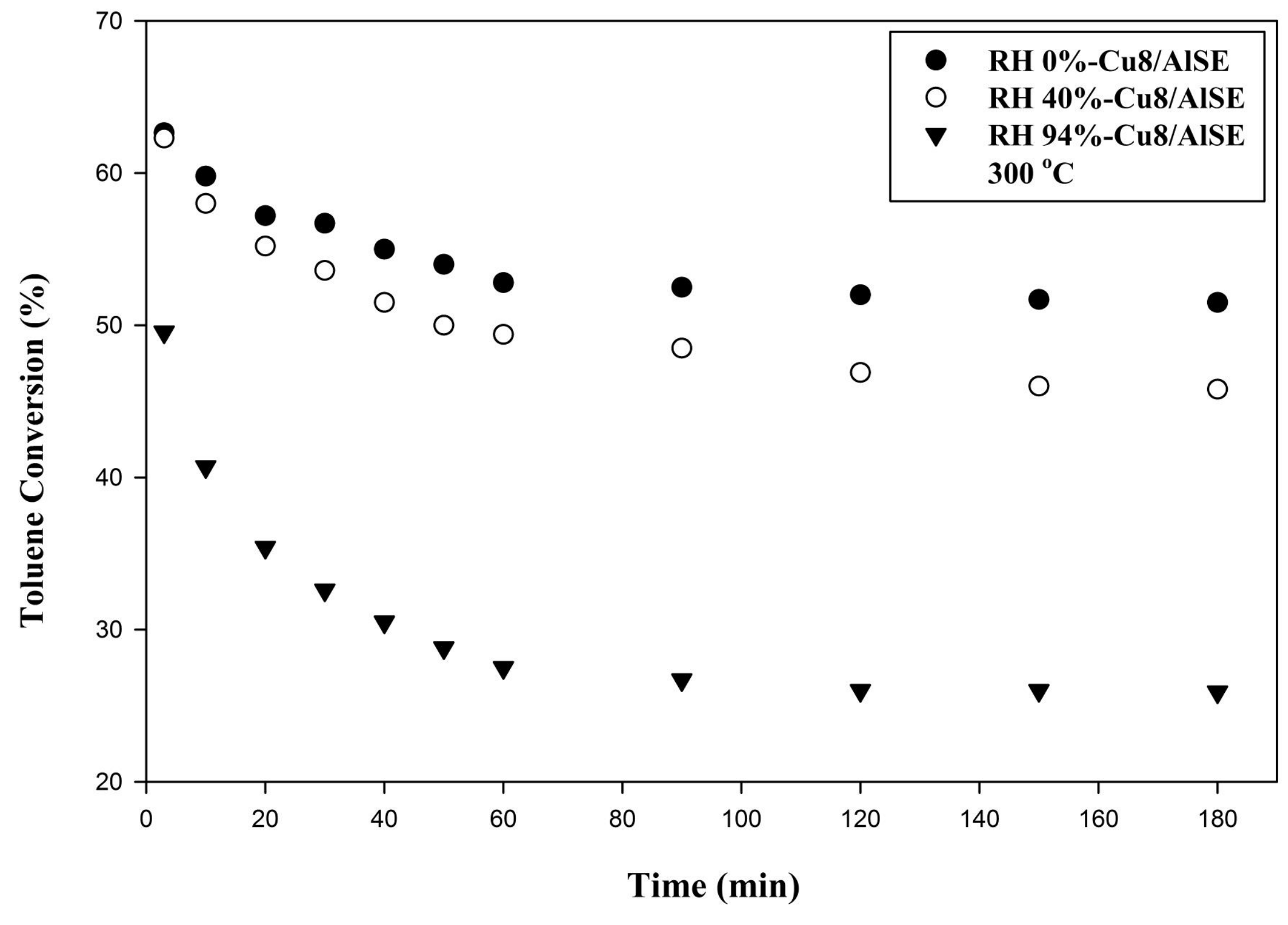




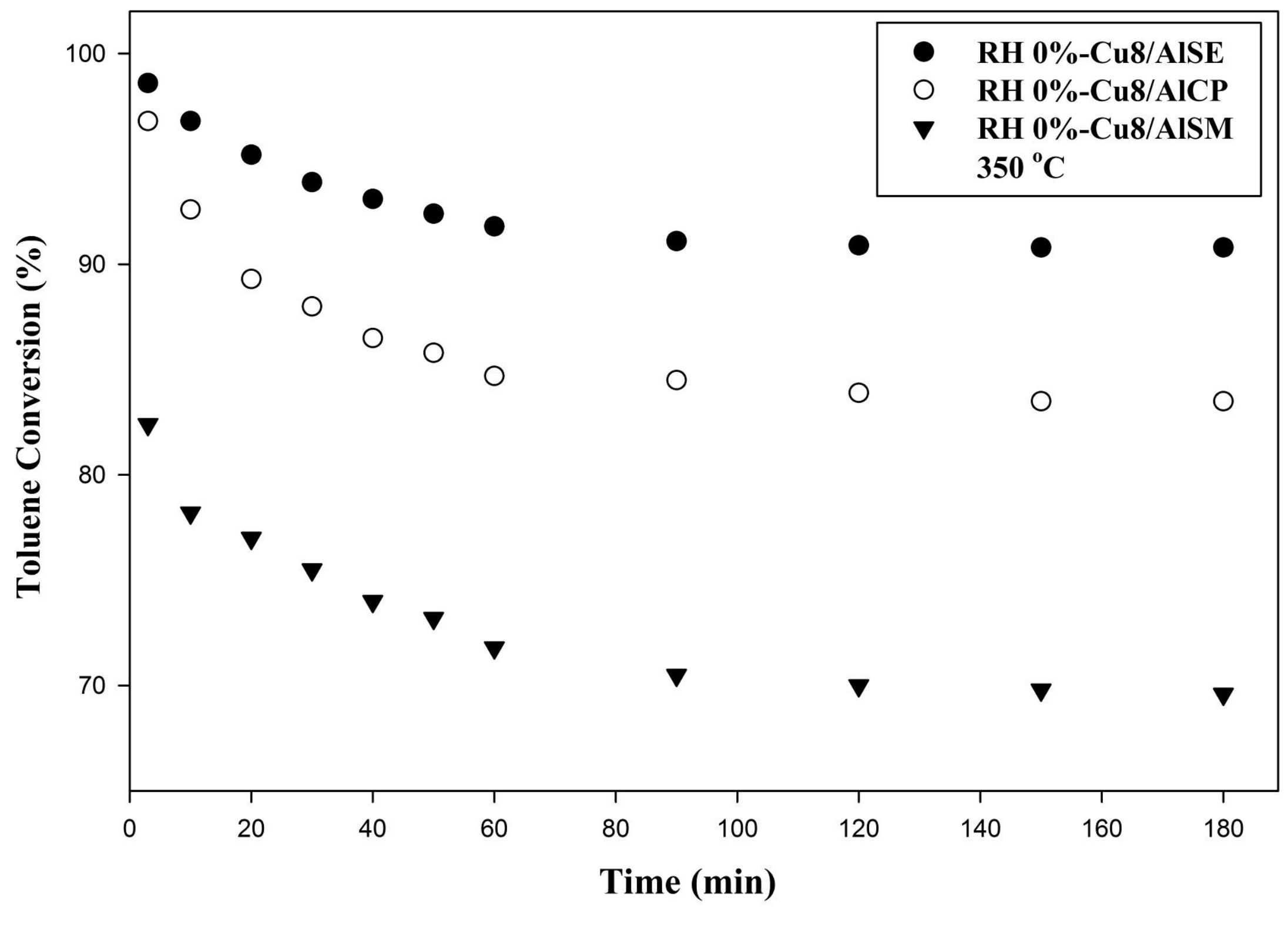




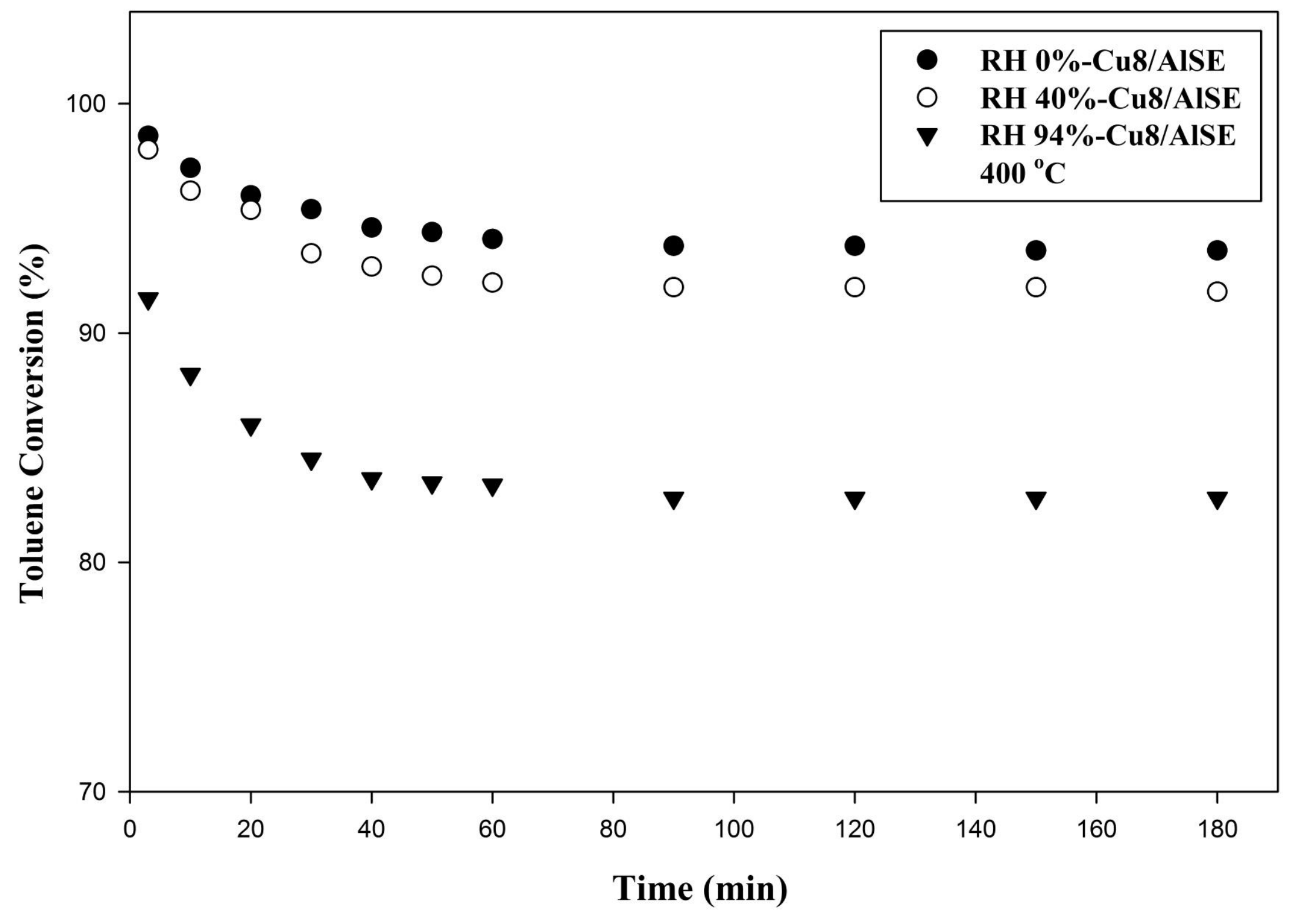




\section{Figure 17.}

Total oxidation of toluene over the prepared catalysts at feed relative humidity of $94 \%$ and reaction temperatures of $350^{\circ} \mathrm{C}$ for a) La2-Cu6/AlSE, La4-Cu4/AlSE, La6$\mathrm{Cu}$ /AlSE, and Cu8/AlSE, b) Co2-Cu6/AlSE, Co4-Cu4/AlSE, Co6-Cu2/AlSE, and Cu8/AlSE, and c) Ni2- Cu6/AlSE, Ni4-Cu4/AlSE, Ni6-Cu2/AlSE, and Cu8/AlSE 


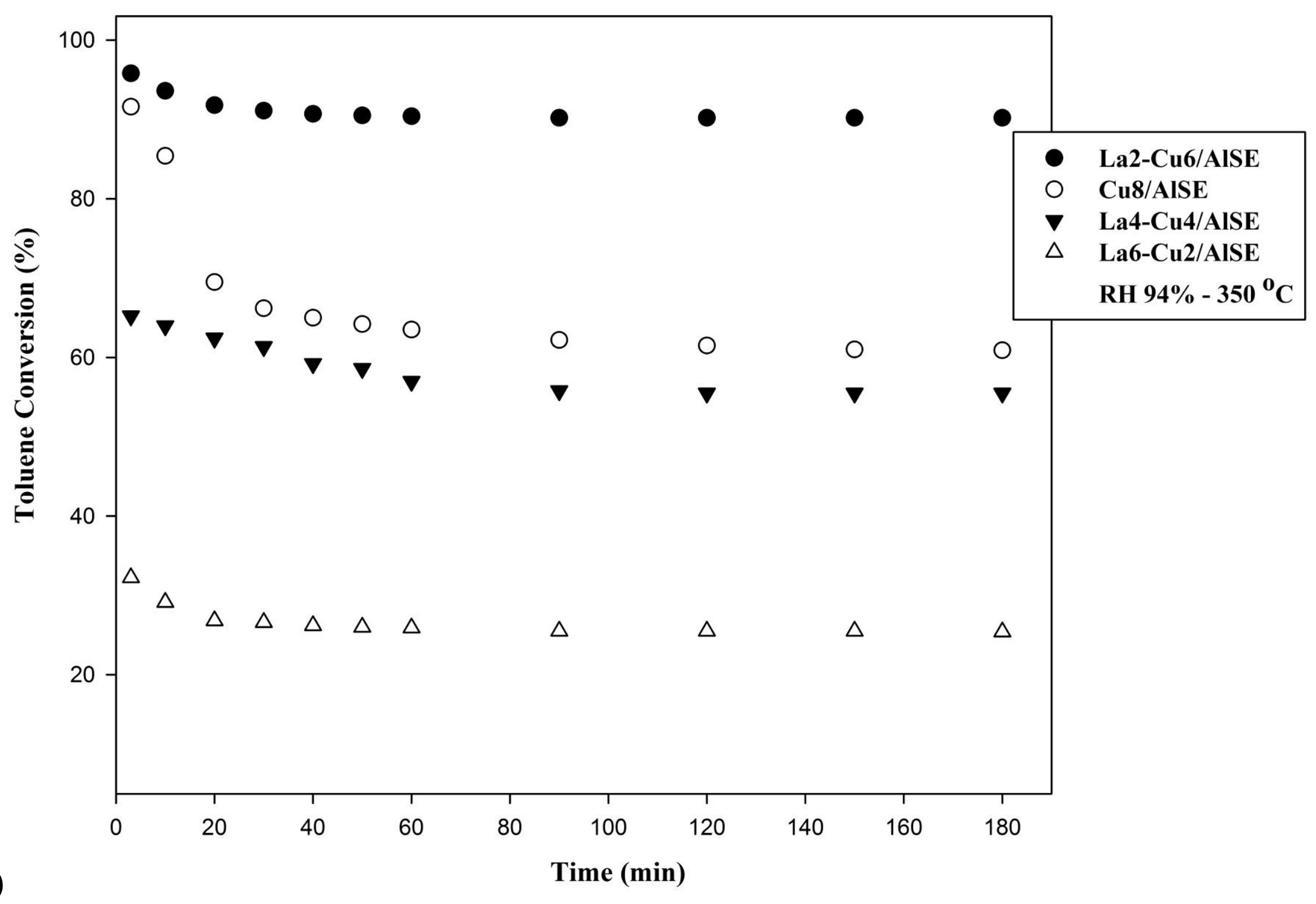




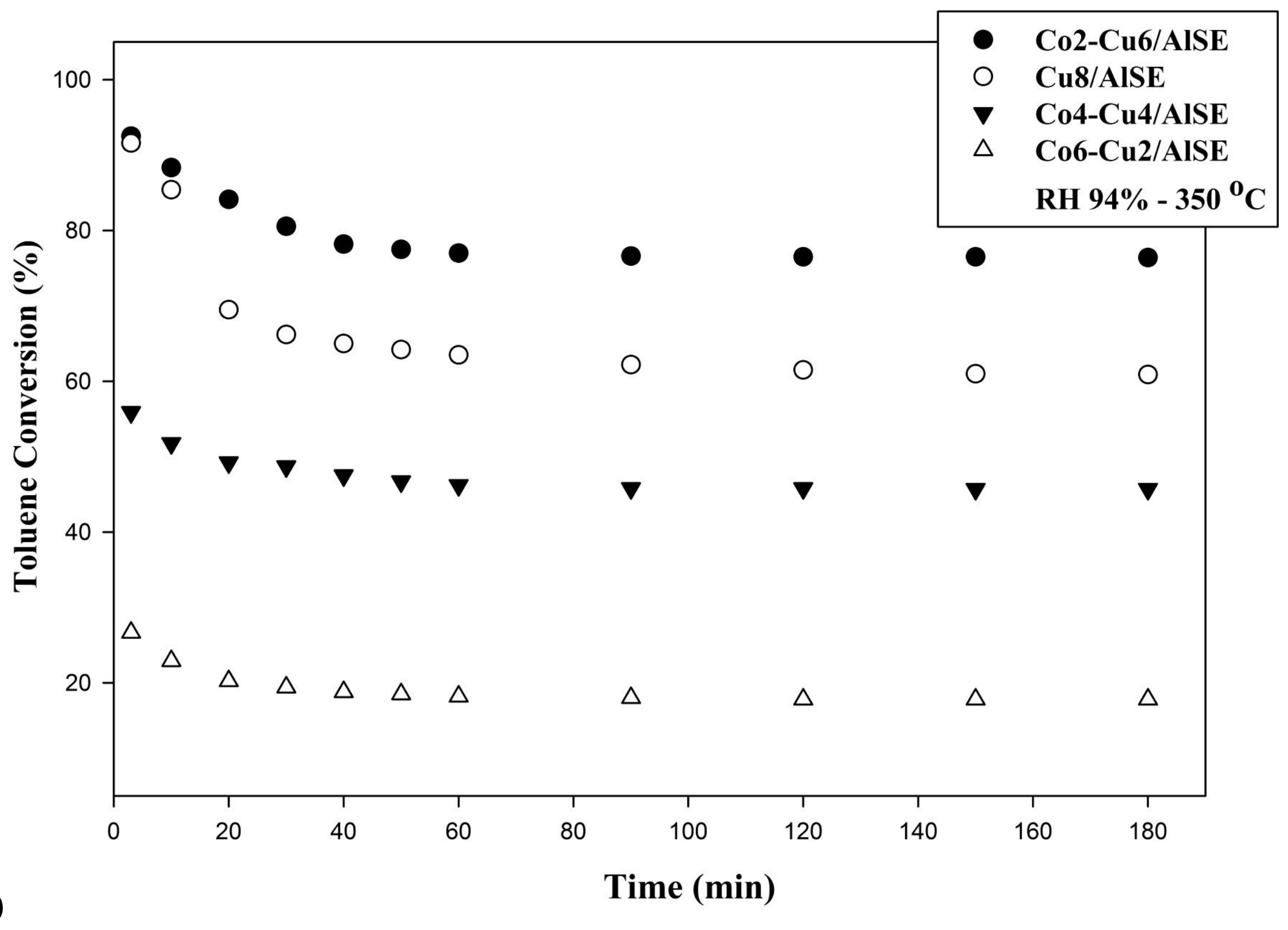




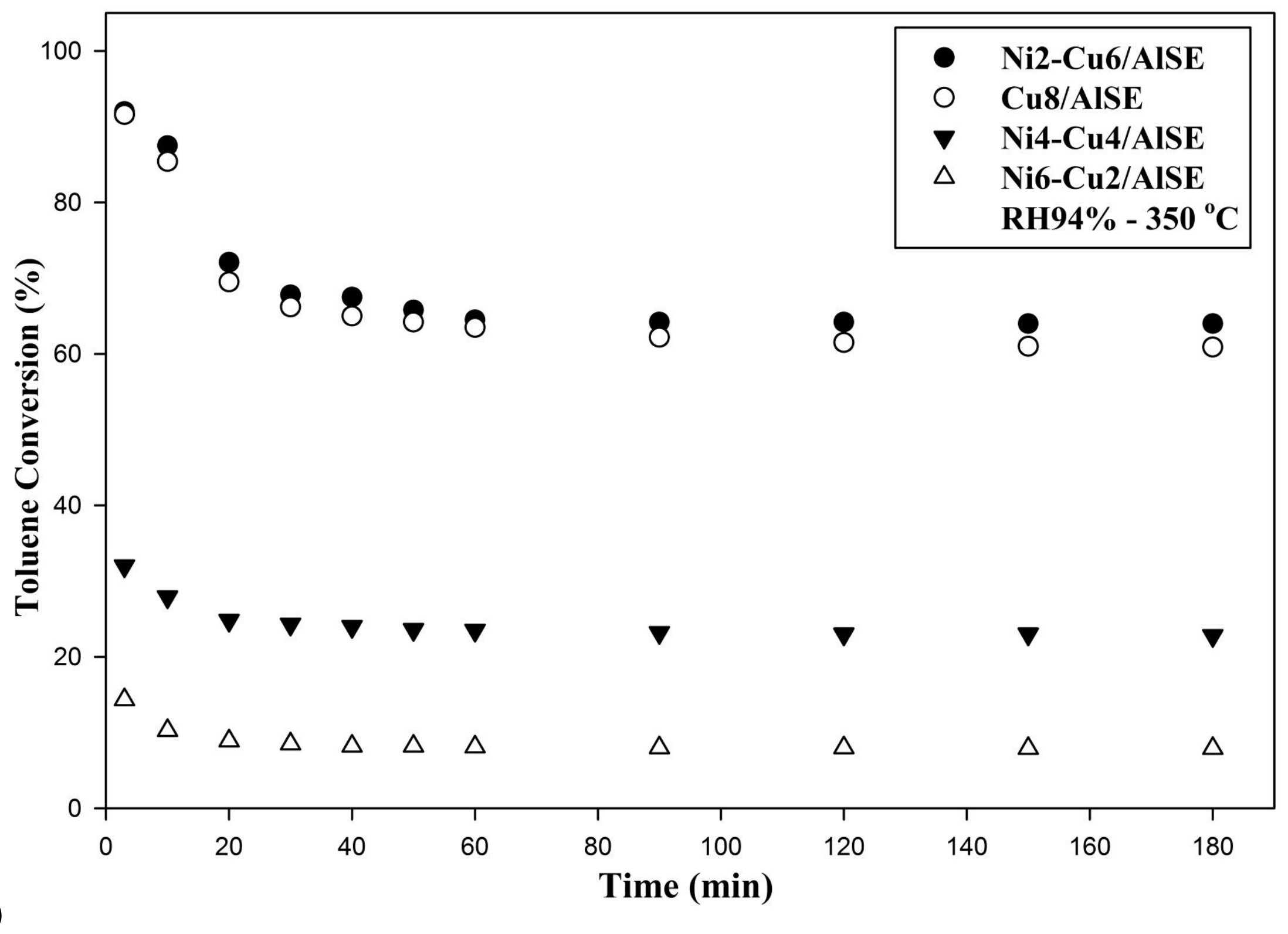




\section{Figure 18.}

Long term performance of La2-Cu6/AlSE for oxidation of toluene at feed relative humidity of $94 \%$ and reaction temperature of $350^{\circ} \mathrm{C}$ 


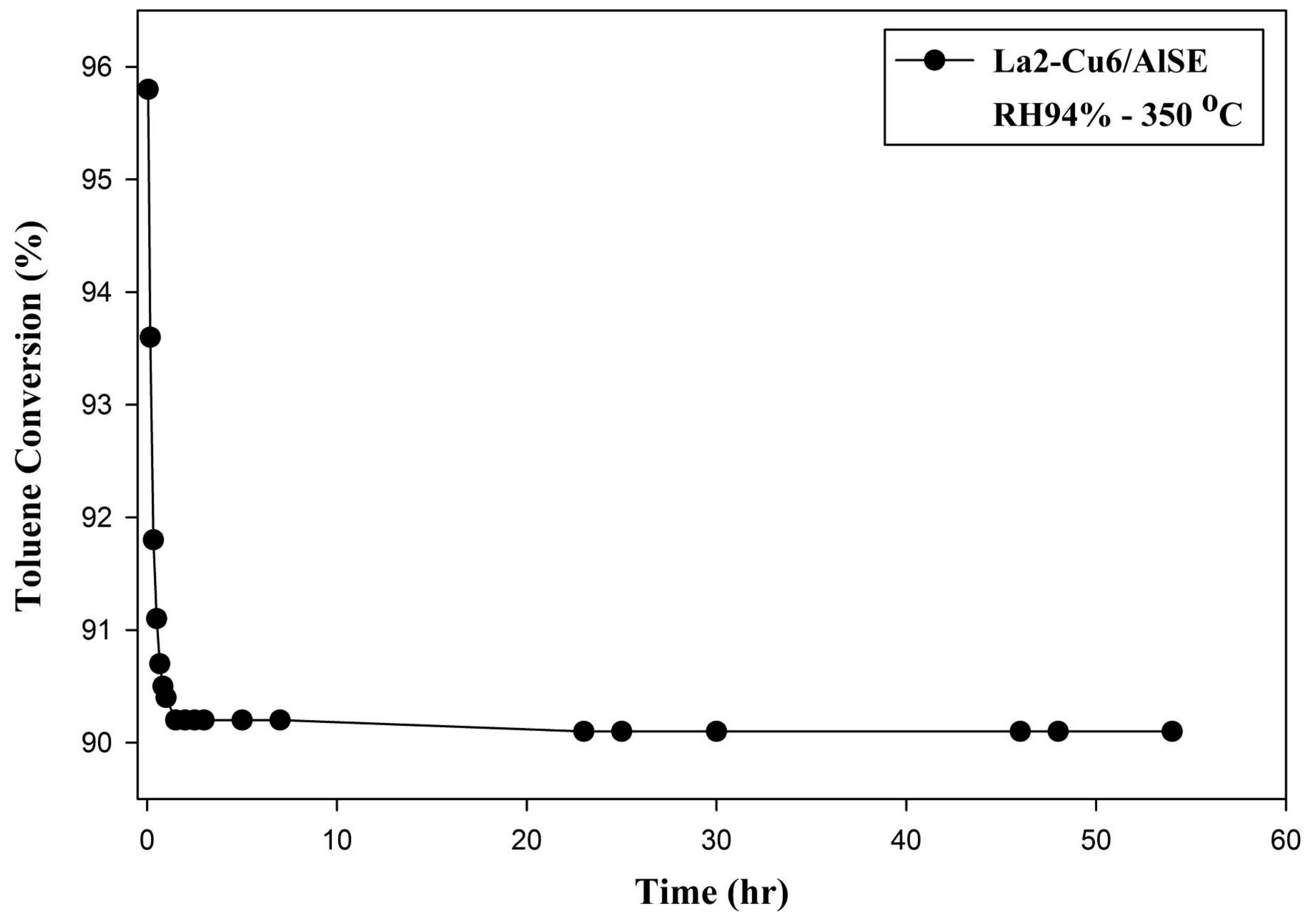

